\title{
Plasticity in visual cortex is disrupted in a mouse model of tauopathy and neurodegeneration
}

\section{Fabio Rodrigues} \\ University College London \\ Joanna Holeniewska \\ University College London \\ Keith Phillips \\ University College London \\ Aman Saleem \\ University College London \\ Samuel Solomon \\ University College London
}

Amalia Papanikolaou ( $\nabla$ amalia.papanikolaou@ucl.ac.uk)

University College London https://orcid.org/0000-0002-0048-6560

\section{Article}

Keywords: neural plasticity, neurodegeneration, tauopathy

Posted Date: June 17th, 2021

DOI: https://doi.org/10.21203/rs.3.rs-602806/v1

License: (c) (i) This work is licensed under a Creative Commons Attribution 4.0 International License.

Read Full License

Version of Record: A version of this preprint was published at Communications Biology on January 20th, 2022. See the published version at https://doi.org/10.1038/s42003-022-03012-9. 


\title{
Plasticity in visual cortex is disrupted in a mouse model of tauopathy and neurodegeneration
}

\author{
Amalia Papanikolaou ${ }^{1,{ }^{*},+}$, Fabio R. Rodrigues ${ }^{1,+}$, Joanna Holeniewska ${ }^{1}$, Keith Phillips ${ }^{2}$, \\ Aman B. Saleem ${ }^{1, \ddagger}$, Samuel G. Solomon ${ }^{1, \ddagger}$ \\ ${ }^{1} \mathrm{UCL}$ Institute of Behavioural Neuroscience, Department of Experimental Psychology, University College \\ London, London, WC1H 0AP; ' ${ }^{E}$ li Lilly, Research and Development, Erl Wood, Surrey, GU20 6PH, United \\ Kingdom.
}

+These authors contributed equally to this work.

期ese authors jointly supervised this work.

*Correspondence: amalia.papanikolaou@ucl.ac.uk

\begin{abstract}
Neurodegeneration is a hallmark of many dementias and thought to underlie a progressive impairment of neural plasticity. Previous work in mouse models of neurodegeneration shows pronounced changes in artificially-induced plasticity in hippocampus, perirhinal and prefrontal cortex. However, it is not known how neurodegeneration disrupts intrinsic forms of brain plasticity. Here we characterised the impact of tau-driven neurodegeneration on a simple form of intrinsic plasticity in the visual system, which allowed us to track plasticity at both long (days) and short (minutes) timescales. We studied $\mathbf{r T g} 4510$ transgenic mice at early stages of neurodegeneration (5 months) and a more advanced stage (8 months). We recorded local field potentials in the primary visual cortex while animals were repeatedly exposed to a stimulus over 9 days. We found that both short- and long-term visual plasticity were already disrupted at early stages of neurodegeneration, and further reduced in older animals, such that it was abolished in mice expressing mutant tau. Additionally, visually evoked behaviours were disrupted in both younger and older mice expressing mutant tau. Our results show that visual cortical plasticity and visually evoked behaviours are disrupted in the rTg4510 model of tauopathy. This simple measure of plasticity may help understand how neurodegeneration disrupts neural circuits, and offers a translatable platform for detection and tracking of the disease.
\end{abstract}

\section{Introduction}

Neurodegenerative diseases are known to affect neural plasticity and memory. Previous research has identified cellular and circuit deficits in higher-order brain areas such as the entorhinal and hippocampal cortices, which may underlie the deficits in memory function. For example, in vitro measurements show artificially-induced (usually electrical) long term potentiation (LTP) is reduced in the hippocampus and frontal cortex in mouse models of Alzheimer's Disease ${ }^{1-4}$. In vivo measurements in these mouse models also show disruption of artificially-induced LTP 5,6 . The complex connections and functions of the hippocampus and frontal cortex, however, make it difficult to design experiments that measure plasticity in these areas without using artificial stimulation. How degeneration disrupts intrinsic forms of brain plasticity is, therefore, largely unknown.

Plasticity and neurodegeneration can occur in all neural circuits, even those involved in simpler brain functions. For example, degeneration is found in the primary visual cortex as well as the hippocampal formation in most transgenic mouse models of degeneration. The visual cortex potentially provides a simpler 
and better-understood model for investigating the functional consequences of neurodegenerative diseases, because there are well established paradigms for studying intrinsic plasticity in the visual cortex, and its behavioural correlates. But which, if any, forms of visual cortical plasticity are impaired in mouse models of neurodegeneration is not known. Indeed, it is not yet clear that degeneration in visual cortex is accompanied by any functional changes. The characteristic orientation selectivity of neurons in V1 is preserved, even in late stages of degeneration, in mice that overexpress amyloid- $\beta$ precursor protein (APP) ${ }^{7,8}$, and in the widely used and well-characterised rTg4510 mouse model of tauopathy9 .

We measured visual plasticity in the $\mathrm{rTg} 4510$ mouse model ${ }^{10,11}$. The $\mathrm{rTg} 4510$ mice develop progressive neurofibrillary tangles (NFT), neuronal loss and concomitant cognitive deficits ${ }^{10,12}$. High levels of mutant tau emerge in the hippocampus and neocortex (including visual cortex) between 2 and 4 months of age, and NFT are present by 4.5 months in hippocampus and 7-8 months in neocortex ${ }^{10,11}$. Cortical cell loss occurs slightly later, at about 8.5 months $^{13}$. We therefore studied animals at early stages of neurodegeneration $(\sim 5$ months old) and at a more advanced stage where clear degeneration has taken place in the cortex $(\sim 8$ months). To characterise intrinsic plasticity, we studied responses to repeated presentation of a simple visual pattern. Over several minutes, repeated presentation of a visual pattern usually suppresses visual cortical responses to that pattern, a classical effect of sensory adaptation ${ }^{14,15}$. Over several days, however, repeated presentation of a visual pattern can instead increase response, involving a sleep-dependent process called stimulus response potentiation, or SRP16-18. SRP is a form of long-term plasticity that resembles canonical LTP, with which it shares mechanisms ${ }^{18}$.

We found that basic visual evoked responses were largely unaffected, even at late stages of neurodegeneration, consistent with previous reports ${ }^{9}$. However, both short- (intra-day) and long-term (interday) visual plasticity were disrupted, even at early stages of neurodegeneration. Both timescales of visual plasticity were further impaired in older animals, such that they were abolished at later stages of neurodegeneration. Additionally, we found that innate visually evoked behaviours were impaired in mice expressing the mutant tau. Our results therefore show that there are substantial changes in intrinsic visual cortical plasticity and in visual behaviour even early in the progression of degeneration in rTg4510 mice. The visual cortex may therefore provide a useful platform to study the impact of neurodegenerative disease on neural plasticity.

\section{Results}

43 We measured the local field potential (LFP) from the primary visual cortex (V1) of $50 \mathrm{rTg} 4510$ mice, using

electrodes targeted to layer 4. Half of the mice were placed on a doxycycline diet from 2 months of age, to suppress the expression of mutant tau (referred as Tau-) $)^{11,13,19}$. We used different animals to study early stages of neurodegeneration ( $\sim 5$ months) and more advanced stages ( 8 months) (Fig. 1A). Mice fed a normal diet, and therefore continuing to express the mutant tau (referred as Tau+) showed increased tau pathology ( $F=23.61, p=10^{-4}$, two-way ANOVA, Fig. 1B, Fig. S1) and a significant reduction in the overall brain weight compared to Tau- animals $(F=5.79, p=0.02$, Fig. $1 B)$.

\section{Visual cortical responses in $r$ Tg4510 mice}

We recorded the visual evoked potential (VEP) in response to a large sinusoidal grating, presented to the monocular visual field by a computer monitor. Mice were head-fixed but free to run on a styrofoam wheel. The contrast of the grating was flipped (reversed) every $0.5 \mathrm{~s}$, and the animals were exposed to 10 blocks of 200 reversals, with 30 s of a grey screen presented between blocks. Each reversal generated a VEP, with an initial negative deflection rapidly followed by a positive one (Fig. 1C). We found that the amplitude of the VEP was similar in Tau- and Tau+ animals, for both 5- and 8-month old animals (Fig. 1D). We calculated the VEP amplitude as the difference between the positive and negative peaks of the VEP signal and found no difference in the average amplitude between genotypes ( $F=0, p=0.95$, two-way ANOVA) or age groups $(F=3.17, p=0.08)$. A Tukey's multiple comparison test showed no significant difference between Tau- and Tau+ VEP amplitudes at 5 months (Tau- $5 \mathrm{~m}: 165 \pm 11$, Tau+ $5 \mathrm{~m}: 184 \pm 16$, mean $\pm S E M, p=0.85$ ) or 8 months (Tau- 8m: 155 \pm 19 , Tau $+8 \mathrm{~m}: 134 \pm 16$, mean \pm SEM, $p=0.79$ ). At 8 months, the VEP signal reduced slightly for Tau+ mice compared to their 5-month old counterparts (Fig. 1E), but the effect did not reach significance 
animals. Our results therefore show that basic visual cortical responses are largely preserved in the rTg4510

A

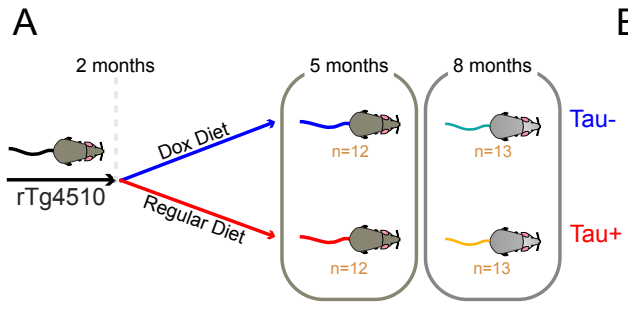

C
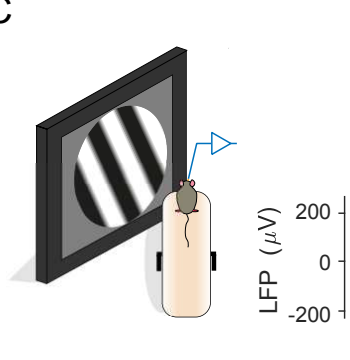

Grey Screen

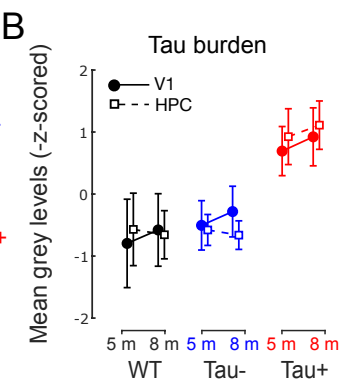

WT Tau- Tau+

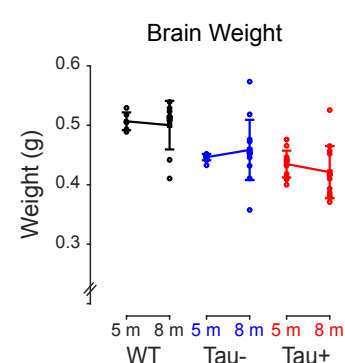

WT Tau- Tau+

Stimulus

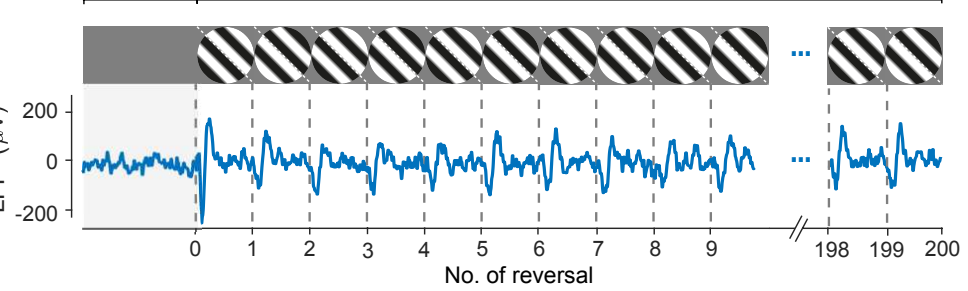

D

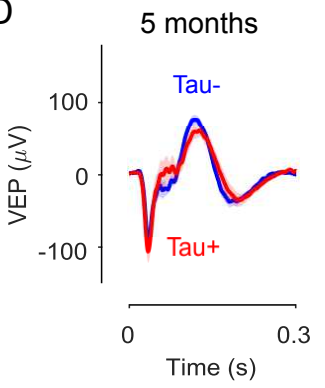

8 months

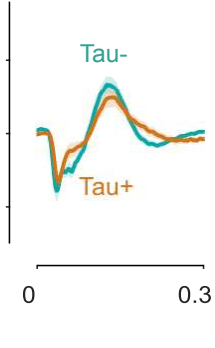

E

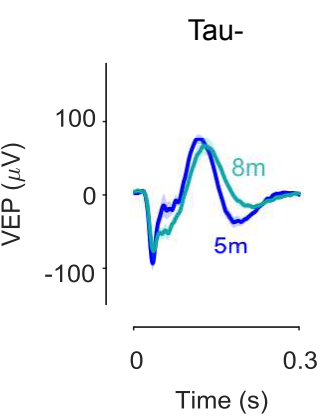

Tau+

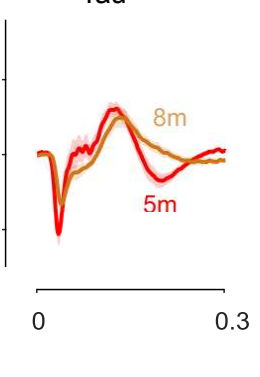

Figure 1 - Visual evoked responses in the primary visual cortex are largely preserved in neurodegeneration. A. At 2 months of age, half the $\mathrm{rTg} 4510$ mice were treated with and fed doxycycline (Dox) to suppress the expression of the transgene and arrest further accumulation of Tau. B. Left, comparison of average tau burden for each group of animals calculated as the mean grey levels within selected regions of interest in V1 and hippocampus (HPC) (Methods). Data are represented as mean $\pm 2 *$ SEM. Right, comparison of overall brain weights at 5 months $(5 \mathrm{~m})$ and 8 months $(8 \mathrm{~m})$ timepoints. Data are represented as mean \pm SEM. C. Mice were head-fixed and allowed to run on a styrofoam wheel. After $5 \mathrm{~min}$ exposure to a homogenous grey screen, a full-screen, contrast reversing, sinusoidal grating pattern was presented to the left monocular visual field. The grating reversed every $0.5 \mathrm{~s}$, and the animal was exposed to 10 blocks of 200 continuous reversals, with 30 s presentation of a grey screen between blocks. We recorded the local field potential (LFP) in the right primary visual cortex (V1). The LFP trace shown is the average time course across 10 stimulus blocks on one day in one Tau- mouse. Each reversal generated a visual evoked potential (VEP), characterized by an initial negative deflection and subsequent positive one. D. Average VEP responses on the first day of recording for Tau- and Tau+ animals at 5 months (left) and 8 months (right). Shaded area represents the SEM. There was no significant difference in the size and shape of the VEPs between groups at either age ( $p=0.95$, two-way ANOVA). E. Average VEP responses for Tau(left) and Tau+ (right) mice obtained at 5 months and 8 months of age. VEPs were slightly reduced, and more sustained, for both Tau- and Tau+ mice at 8 months. 
We hypothesised that plasticity is more likely to be affected than basic visual response at early stages of neurodegeneration. Stimulus-Response Potentiation (SRP) is a form of intrinsic cortical plasticity induced by repeated exposure to a visual stimulus over several days that can be measured in the VEP of mouse $\mathrm{V} 1^{16}$. To measure SRP in these animals we obtained VEP measurements from 24 5-month old rTg4510 mice (12 Tau- and $12 \mathrm{Tau}+$ ) while they were exposed to a grating of one orientation (either $45^{\circ}$ or $-45^{\circ}$ from vertical) over 9 days (thus becoming a familiar stimulus). On the first and last day of recordings (day 1 and day 9 ), five blocks of this familiar stimulus orientation were interleaved with five blocks of the orthogonal orientation (unfamiliar stimulus). On days 2-8, 10 blocks of the familiar stimulus were presented (Fig. 2A).
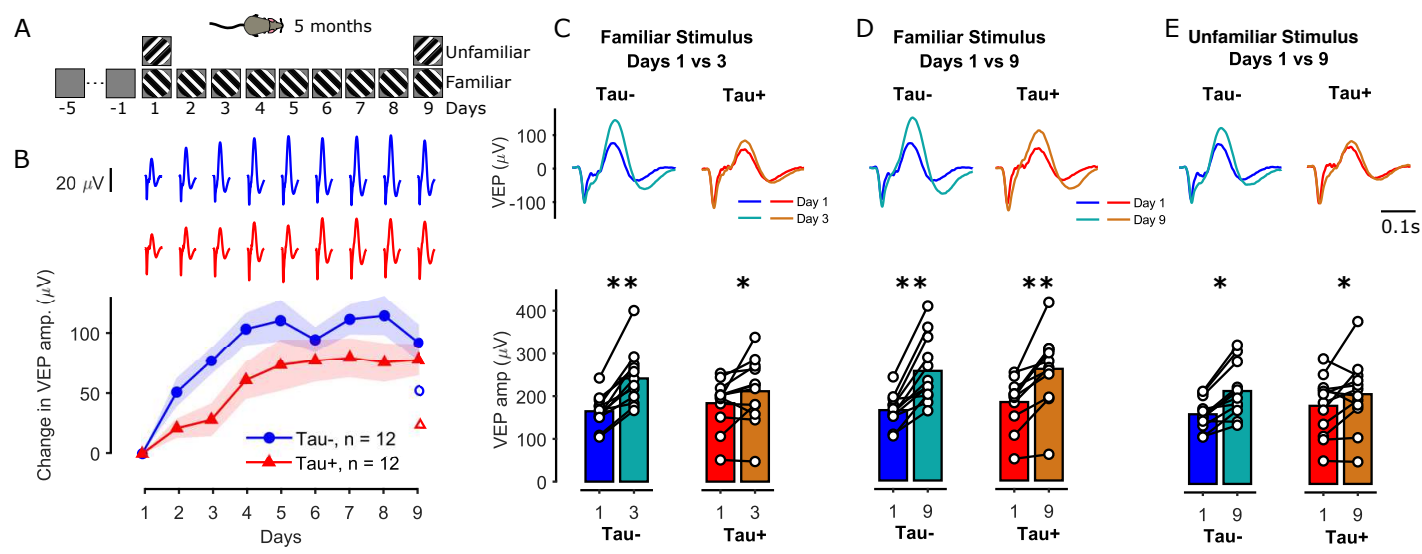

Figure 2. Visual plasticity is reduced at early stages of tauopathy. A. Mice (5 months old) were exposed to a grating of one orientation (either $45^{\circ}$ or $-45^{\circ}$; 'familiar' stimulus) for 9 days. On the first and last day of recordings, five blocks of this familiar stimulus were interleaved with five blocks of a grating ('unfamiliar') whose orientation was orthogonal to the familiar grating. On days 2-8, 10 blocks of the familiar stimulus were presented. B. Change in the VEP amplitude from day 1 for the familiar stimulus for Tau- (blue) and Tau+ (red) animals over the course of days. Tau+ mice showed a slower potentiation of the LFP signal compared to Tau- animals. The open symbols on the right show the change in VEP amplitude in response to the unfamiliar stimulus on day 9. C-E. Comparison of average VEPs (top) and VEP amplitudes (bottom) between: days 1 vs 3 for the familiar stimulus (C), days 1 vs 9 for the familiar stimulus (D), and days 1 vs 9 for the unfamiliar stimulus (E).

We found strong potentiation of the VEP signal in Tau- animals, and reduced and slower potentiation in Tau+ animals (Fig. 2). The VEP amplitude for Tau- mice reached a plateau around day 3-4 (Fig. 2B) while Tau+ mice showed a slower increase in the VEP amplitude, reaching a plateau around day 5-6. For example, the VEP amplitude of Tau- mice was $77 \pm 11 \mu \mathrm{V}$ larger on day 3 than it was on day 1 (Fig. 2B,C; $p=3.1^{*} 10^{-6}$, repeated measures ANOVA, Tukey's pairwise comparison), while Tau+ animals showed only a moderate increase by day $3(28 \pm 13 \mu \mathrm{V}, \mathrm{p}=0.03)$. By day 9 , VEP amplitude had increased substantially for both groups of mice (Fig. 2D, Tau-: $92 \pm 15 \mu \mathrm{V}, \mathrm{p}=1.5^{\star} 10^{-6}$, Tau+: $78 \pm 13, \mathrm{p}=1.6^{\star} 10^{-5}$ ). Repeated measures ANOVA on days 1 and 3 revealed a significant day by phenotype interaction $(F=7.75, p=0.01)$, while the same comparisons for days 1 and 9 showed a significant effect of day $\left(F=72.1, p=2.1^{*} 10^{-8}\right)$ but no interaction $(F=0.5, p=0.48)$. The slower growth in VEP amplitude in Tau+ animals was mainly due to slower changes in the positive deflection of the VEP signal in Tau+ animals (Fig. S2b). The negative deflection of the VEP was slightly larger in Tau+ mice than in Tau- mice, and increased at a similar rate in both groups (Fig. S2c).

Repeated exposure to the familiar stimulus had less impact on VEP response to the unfamiliar stimulus (which was shown only on day 1 and day 9), for both groups (Fig. 2E, Tau-: unfamiliar VEP amp. change $=54 \pm 10, p=0.2^{*} 10^{-3}$, Tau+: $15 \pm 27, p=0.03$, Tukey's test). In fact, growth in VEP responses to the unfamiliar stimulus on day 9 was comparable to growth in VEPs to the familiar stimulus on day 2 (Fig. 2B, Tau-: day 2 familiar VEP amp. change $=51 \pm 12, p=0.89$, Tau+: $21 \pm 8, p=0.72$ ). Together these data suggest that the amplitude but not stimulus selectivity of response potentiation is affected in Tau+ animals.

Overall our results suggest that visual cortical plasticity is disrupted even at this early stage of neurodegeneration. 
124 We showed that visual plasticity is affected even at early stages of neurodegeneration in rTg4510 mice. We then asked whether functional deficits increase with age by examining visual cortical plasticity in 8-month old rTg4510 mice. We obtained LFP responses from 26 8-month old transgenic mice (13 Tau- and 13 Tau+) using the same visual paradigm described above (Fig. 3A).

Visual plasticity was reduced in older animals (Fig. 3). By day 9, the VEPs of Tau- mice showed a small but significant increase relative to day 1 (Fig. $3 C$, VEP amp. change $=39 \pm 20 \mu \mathrm{V}, \mathrm{p}=0.02$ ), while Tau+ animals showed minimal change $(13 \pm 9, p=0.41)$. No significant change was observed for the unfamiliar stimulus in either group (Fig. 3D, Tau-: VEP amp. change $=25 \pm 16, p=0.72$, Tau+: $6 \pm 9, p=0.63$ ). Our results suggest that there may be a combined effect of mutant tau expression and age leading to a complete disruption of visual cortical plasticity.
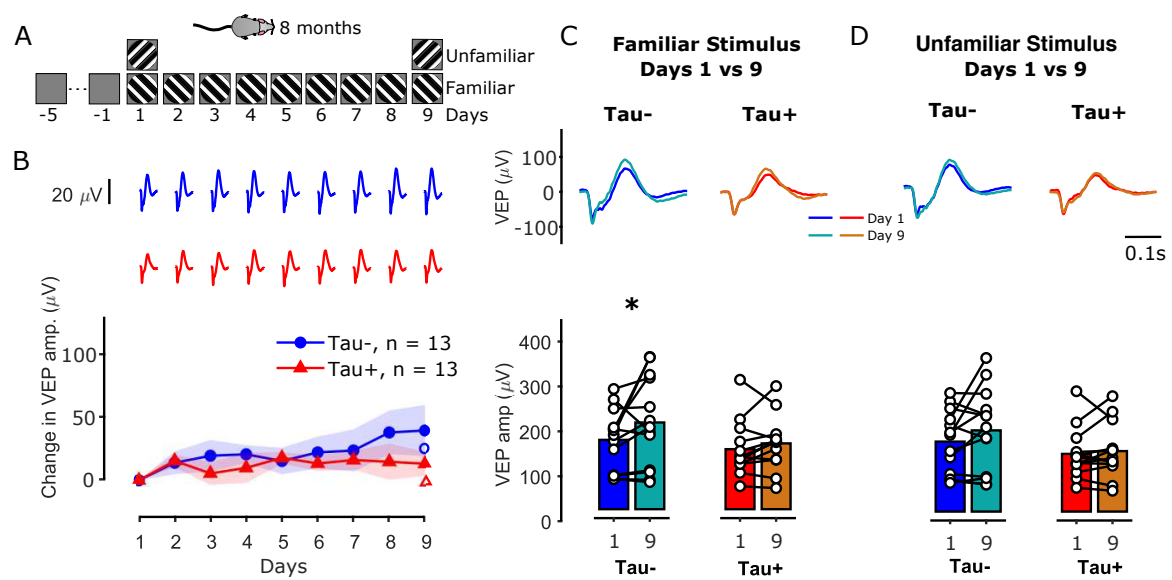

Figure 3. Visual plasticity diminishes with age. A. Mice ( 8 months old) were exposed to a grating of one orientation (either $45^{\circ}$ or $-45^{\circ}$; 'familiar' stimulus) for 9 days (same as in Fig. $2 \mathrm{~A}$ ). B. Difference in the VEP amplitude from day 1 for the familiar stimulus for Tau- (blue) and Tau+ (red) animals over the course of days. 8-month old Tau- mice showed a smaller potentiation of the LFP signal compared to 5-month old mice (Fig. 2B). Tau+ mice showed no significant potentiation. The open symbols on the right show the change in VEP amplitude in response to the unfamiliar stimulus on day 9. C-D. Comparison of average VEPs (top) and VEP amplitudes (bottom) between: days 1 vs 9 for the familiar stimulus (C), and days 1 vs 9 for the unfamiliar stimulus (D).

\section{Similar response potentiation in Tau- and WT mice}

Tau- mice were fed with a doxycycline diet to suppress mutant tau transgene expression from the age of 2 months. It is possible however that expression of mutant tau up to the age of 2 months, or a continued effect thereafter, could lead to functional deficits in these animals. For example, the reduced visual plasticity observed in 8-month old compared to 5-month old Tau- mice might be a pathological effect initiated by early tauopathy. We therefore compared the visual responses of Tau- mice with responses obtained from wild type (WT) littermates.

At 5 months, WT mice showed similar VEP potentiation to the Tau- mice (Fig. S3A). By day 3, WTs showed a significant increase in the VEP amplitude for the familiar stimulus relative to day 1 (VEP amp. change $=57 \pm 11 \mu \mathrm{V}, p=0.005$ ), similar to Tau- mice (Fig. S3A). At 8 months, the VEP amplitude was on average larger in WT compared to Tau- mice $(F=0.92, p=0.35$, one-way ANOVA). As for Tau- mice, however, 8-month old WT mice showed reduced and slower potentiation of the LFP signal for the familiar stimulus compared to 5-month old mice (Fig. S3B). This suggests that the reduction in SRP at 8 months is largely an effect of age and not neurodegeneration in Tau- mice.

\section{Coincident changes in short-term visual plasticity}

We have shown that long-term visual plasticity is disrupted in rTg4510 mice using a simple and wellestablished visual paradigm. Although SRP has been conventionally used as a measure of plasticity across 
days, it can also be used to measure changes within a day, or within a block (adaptation). We therefore asked if there were also disruptions in plasticity at these shorter timescales. We analysed how responses changed within each day. We considered days 2-8 where only the familiar stimulus was presented (Fig. 4A). Responses gradually increased over the course of the first block of visual stimuli in all animals. In subsequent blocks, the amplitude of the VEPs usually reduced over the course of each block, consistent with classic sensory adaptation effects (Fig. 4B). We therefore focussed our analyses on the second and subsequent stimulation blocks. To quantify adaptation's effects, we fitted a decaying exponential function to the withinblock time course of the VEP amplitude. We obtained the within-block time-course by subtracting the steadystate VEP amplitude (the average of the last 100 reversals) from each block, and then averaging across blocks. We first averaged VEP responses across blocks 2-10 and found smaller adaptation effects in Tau+ than Tau- mice (Fig. 4C left panel; Tau-: $34 \pm 29 \mu \mathrm{V}$, Tau+: $19 \pm 40$, p=0.0075, t-test). Adaptation effects were reduced in 8-month old Tau- mice compared to 5-month olds, and they were completely abolished in 8-month Tau+ mice (Fig. 4E,F; Tau-: 11 \pm 21 , Tau+: $-2 \pm 23, p=0.0001$ ).

A
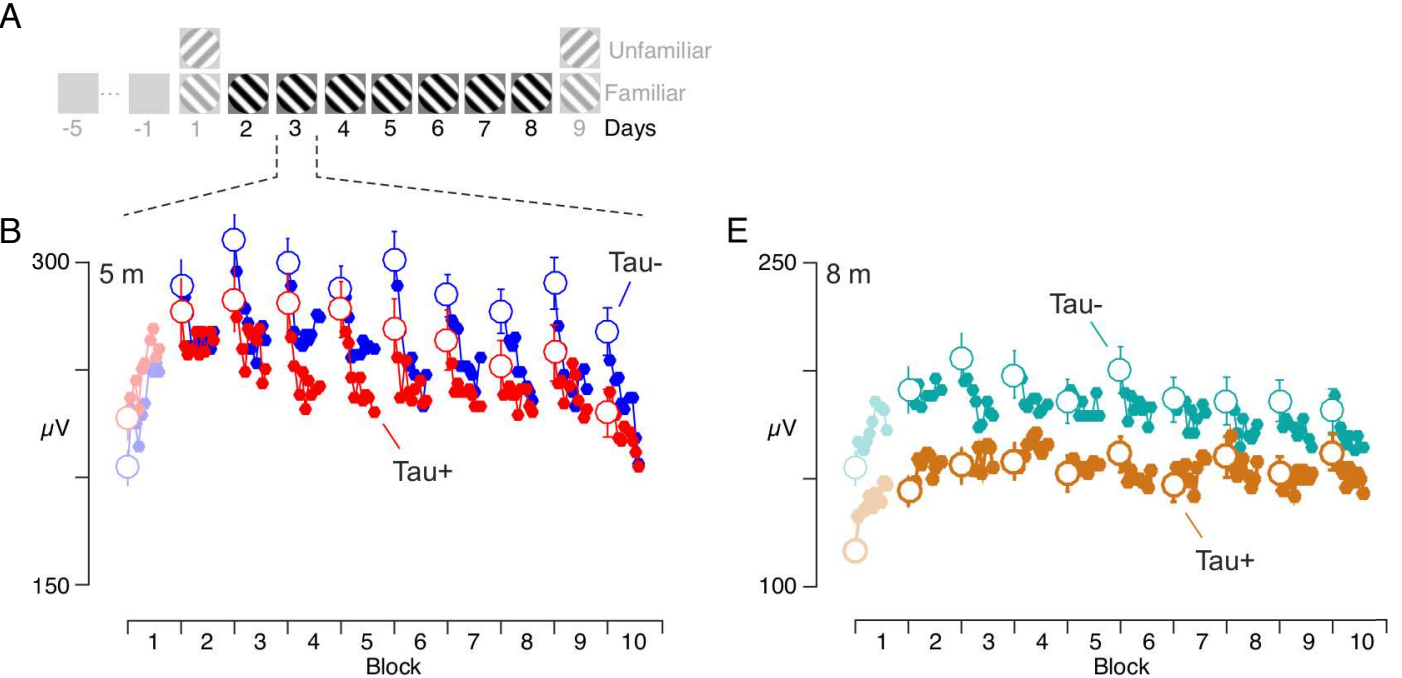

C
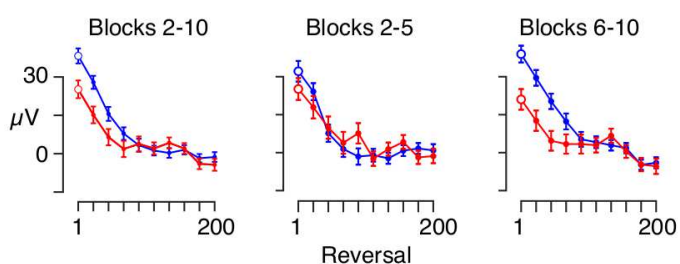

$\mathrm{F}$

D
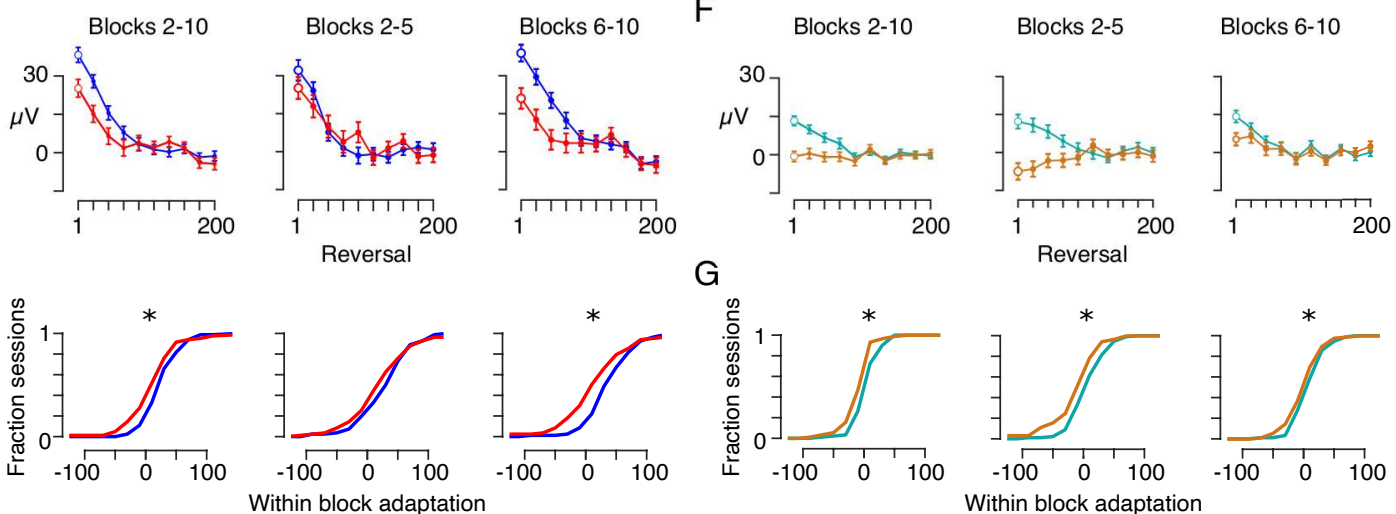

Figure 4: Short-term visual plasticity is reduced in rTg4510 mice expressing mutant tau. A. We measured intra-day effects on experimental days 2-8. On each day 10 blocks of the familiar stimulus were presented, each consisting of 200 phase reversals and separated by 30 s of a homogenous grey screen. B. Average VEP amplitude as a function of block number for 5-month old Tau- (blue) and Tau+ (red) mice. The VEP amplitude was calculated from non-overlapping averages of 20 reversals. With the notable exception of the first block, VEPs showed a reduction of responses within each block, consistent with classic sensory adaptation effects. C. Average VEP responses for blocks 2-10 (Left) and separately for blocks 2-5 (Middle) blocks 6-10 (Right) on days 2-8, for Tau- and Tau+ animals. VEP amplitudes within each block were normalised by subtracting the average VEP amplitude of the last 100 reversals for that block. The within block amplitudes (at a resolution of 20 reversals) were then fit with a decaying exponential function of fixed $\mathrm{T}=8.4$ reversals (see Methods), and the amplitude of the exponential was extracted. D. Cumulative histograms of these fitted amplitudes for Tau+ (solid red) and Tau- (dotted blue) mice, normalised by the total 
number of sessions for each cohort. At 5 months, Tau+ animals showed reduced within-block adaptation compared to Tau- mice for blocks 6-10, but not for blocks 2-5. E-F. Same as B-D for 8-month old animals. The within-block adaptation is reduced for Tau- mice at 8 months compared to 5 months. Tau+ mice showed no suppressive adaptation effect for either early or late blocks.

The disruption to adaptation effects in 5-month old Tau+ mice was more apparent in later blocks than early blocks (Fig 4C). We calculated average VEP amplitudes separately for early blocks (2-5) and late blocks (610), and estimated adaptation's effect for both sets for each recording day in each animal (Fig. 4D). Tau+ animals showed less adaptation effects that Tau- mice in blocks 6-10 (Tau-: $47 \pm 43 \mu \mathrm{V}$, Tau+: $23 \pm 58$, $p=0.0025$, t-test), but similar adaptation effects in blocks $2-5$ (Tau-: $36 \pm 42 \mu \mathrm{V}, \mathrm{Tau}+: 28 \pm 53, p=0.328$, t-test). Within-block adaptation effects were absent in 8-month Tau+ mice in both early and late blocks (Fig. 4F,G; blocks 2-5: Tau-: 14 \pm 29 , Tau+: $-7 \pm 39, p=0$; blocks 6-10: Tau-: $16 \pm 25$, Tau+: $7 \pm 27, p=0.0153$, t-test). We conclude that adaptation effects are disrupted early in neurodegeneration, and abolished at later stages.

We found that, unlike Tau- animals, 8-month old WTs showed similar within block adaptation effects to 5month old WTs, suggesting that while long term plasticity is reduced in older WT animals, short term plasticity is not (Fig. S4, Fig. 4; 5m WT: $26 \pm 29$; 8m WT: 27 \pm 31 ). Reduced adaptation in older Tau- animals compared to WT may reflect persistent impact of the initial tau accumulation before the onset of the doxycycline treatment (which was started at 2 months), subsequent incomplete suppression of the transgene, or agedependent effects of other genetic disruptions in this mouse model (see Discussion).

\section{Differences in visual plasticity cannot be explained by differences in behavioural state}

Animals were free to run on a foam wheel during the recording session, and we observed epochs of running and variations in pupil size in all animals. Visual cortical responses, as well as responses earlier in the visual pathway, are known to vary with behavioural state as defined by running speed or pupil area ${ }^{20}$. Tau+ rTg4510 mice have also been reported to have abnormal locomotion behaviours ${ }^{19,21,22}$. We therefore wanted to know if the differences observed in visual plasticity between Tau+ and Tau- animals might be explained by differences in behavioural state. We used two approaches to address this. We first refined our analyses by only considering VEP responses during either stationary (speed $<5 \mathrm{~cm} / \mathrm{s}$ ) or running (speed $>$ $5 \mathrm{~cm} / \mathrm{s}$ ) epochs. We next used a model to evaluate the contribution of various parameters to the observed responses.

The VEP signal was smaller during running, in both Tau- and Tau+ mice (Fig. 5A). This reduction was seen across all days and animals (Fig. 5B), and was slightly more pronounced in Tau+ animals. To establish whether the animal's locomotion state was responsible for the differences observed in SRP between groups, we calculated the change in the VEP amplitude from day 1 , but considering only stationary or only running epochs (Fig. 5C; Sup. Fig. S5B). The differences in SRP between Tau- and Tau+ mice were more pronounced when we considered only stationary epochs (Fig. 5C, S5C-D), so differences in running behaviour cannot explain the differences in SRP.

Our analyses show that VEP amplitude is dependent on behavioural state, and varies within a day (Fig 4). These effects might combine to accentuate or mask plasticity across days. To assess the relative impact of time and behaviour on the VEP, we used an elastic net regularization model to predict the VEP amplitude for each animal using Day, Block number, movement Speed and Pupil diameter as regressors. We found Day to have a positive impact on the VEP amplitude, thus predicting the increase in VEP amplitude across days (Fig. 5D). Consistent with our observations on visual plasticity (Fig. 2,3), the impact of Day in predicting the VEP amplitude was greater for Tau- than Tau+ animals, for both 5-month old and 8-month old animals. Similarly, Day had a lower impact at 8 months than 5 months old. Block number had a negligible overall impact in predicting the VEP amplitude compared to the other predictors considered here. Increases in behaviour (defined by pupil diameter and speed) had a negative impact on the VEP amplitude, and this relationship was similar across phenotypes and age groups. In addition, there was no correlation between behaviour and days (Pupil: $r=-0.03$, Speed: $r=0.07$ ) suggesting that behaviour cannot explain the VEP increase across days. Therefore, our results suggest that the VEP potentiation to the familiar stimulus across days is an effect of experience to the visual stimulus and not an effect of changes in the behavioural state of the animals. 

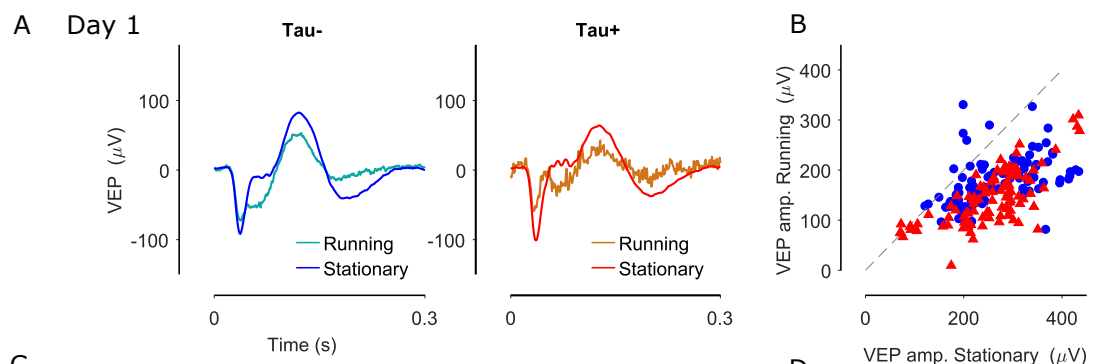

Tau-, $n=12$
Tau+, $n=12$

C

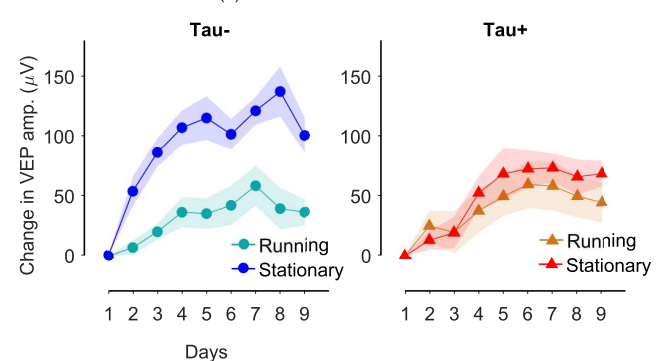

D

VEP amp. Stationary $(\mu \mathrm{V})$

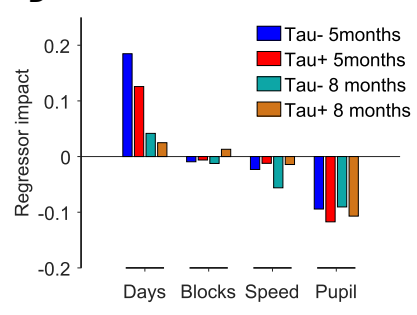

Figure 5: Differences in visual plasticity are not due to differences in behavioural state. A. VEP responses on day 1 for Tau- (blue) and Tau+ (red) 5-month old animals calculated during running (speed $>5 \mathrm{~cm} / \mathrm{s}$ ) and stationary epochs. Running reduces the VEP signal for both groups of mice. B. VEP amplitude during stationary versus running epochs for individual Tau- (blue circles) and Tau+ (red triangles) mice. Each symbol represents the VEP amplitude on one day in one animal. C. Difference in the VEP amplitude from day 1 for the familiar stimulus, for Tau- (left) and Tau+ (right) 5-month old animals, calculated for only stationary or only running epochs. VEP potentiation was evident in both groups of animals for both behavioural states. D. We fitted an elastic net regularization model to predict the VEP amplitude for each animal using days, block number, movement speed and pupil diameter as regressors. We assessed the impact of each regressor in predicting the VEP amplitude as the product between each learned weight and observed variable, divided by the predicted value across the entire regularisation path (of elastic net regression). The panel shows the average regressor impact for each genotype at 5 and 8 months of age.

246 Visual evoked behaviours are reduced in naive Tau+ mice

247 In animals, including mice, an unexpected or unfamiliar visual stimulus usually evokes instinctive behavioural responses. In head-fixed animals who are unable to run, these behavioural responses can include low amplitude muscle movements ('fidgeting'). SRP in the mouse visual cortex has previously been associated with habituation of fidgeting ${ }^{18}$. To establish whether reduced plasticity in Tau+ mice is associated with reduced behavioural responses in our conditions, we assessed the impact of stimulus presentation on pupil diameter and movement speed, both at first exposure to the stimulus, and later when animals were more experienced.

Tau- (and WT) mice showed large dilation of the pupil and an increase in the movement speed at the onset of their very first exposure to the grating stimulus (day 1, block 1; Fig. 6A,B, Fig. S3). These responses quickly habituated, both within the session (Sup. Fig. S6) and over the course of days (Fig. 6C,D). By contrast, Tau+ mice showed no visual evoked behavioural responses to the onset of the stimulus (Fig. 6A,B). These group differences in visual evoked behaviours were clear at both 5 and 8 months of age.

Experienced Tau- animals (days 6-8) showed a small constriction of the pupil in response to the stimulus onset. This constriction was more pronounced in Tau+ animals (Fig. 6C). There was no change in the movement speed for both groups in response to the stimulus, although experienced Tau- mice showed larger average speed compared with the Tau+ mice (Fig. 6D). Overall, our results suggest that visual evoked responses are disrupted even in early stages of neurodegeneration. 
A
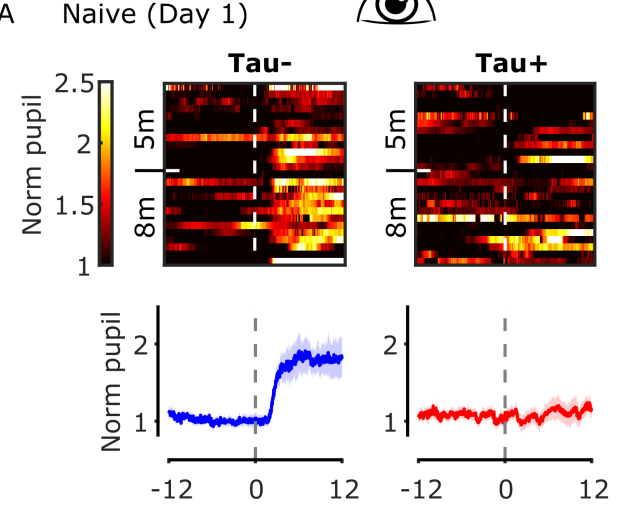

C Experienced (Days 6-8)

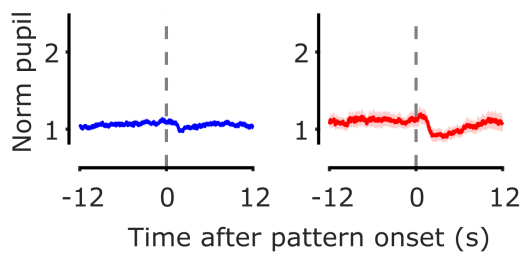

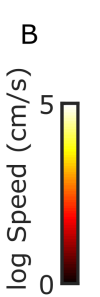
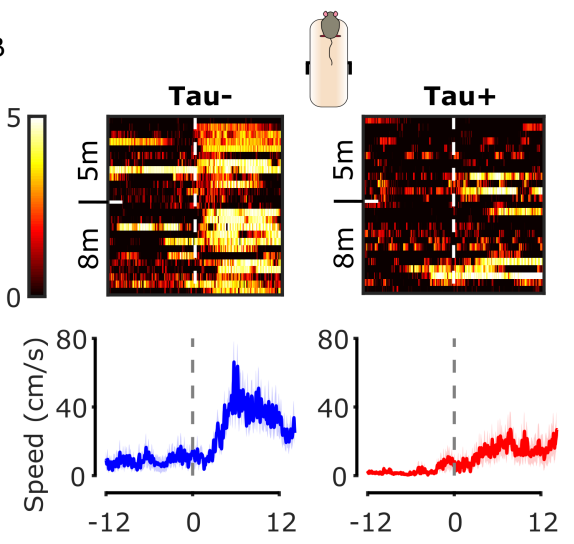

D

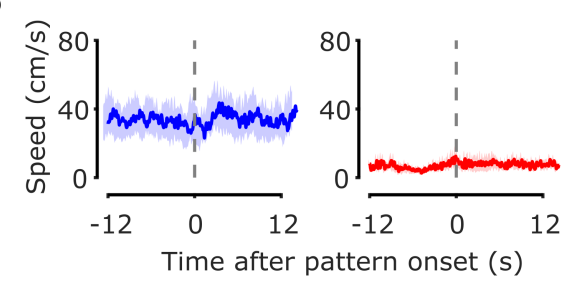

Figure 6: Visual evoked behaviours are reduced in Tau+ mice. A. Top: Images of the normalized pupil responses to the onset of the stimulus of the first block presented for naive animals (day 1; one animal per row). Pupil responses are normalized to the average pupil size during stationary epochs for $12 \mathrm{~s}$ before the stimulus onset where mice were viewing a grey screen. Bottom: Mean $( \pm S E M)$ pupil responses for naive Tau- (blue) and Tau+ (red) animals for both age groups. B. Top: Images of the natural logarithm of movement speed responses to the onset of the stimulus for naive animals (day 1). Bottom: Mean ( \pm SEM) movement speed for naive Tau- (blue) and Tau+ (red) animals for both age groups. C-D. Average normalized pupil responses and average movement speed to the onset of the stimulus for experienced animals (days 6-8). Horizontal dashed lines indicate the stimulus onset.

\section{Discussion}

In this study, we evaluated visual cortical plasticity in the rTg4510 mouse model of neurodegeneration. We measured both short term suppression and long-term potentiation of the visual evoked LFP response in V1, in mice with mutant tau expression (Tau+), or with that expression suppressed (Tau-). We made these measurements at two time points, at an early (5 months), and a more advanced ( 8 months) stage of neurodegeneration ${ }^{10,11,13,23}$, which allowed us to estimate the progression of the pathology and its correlates in cortical plasticity. The results indicate that visual evoked responses are robust in both Tau- and Tau+ mice in both age groups. However, Tau+ animals show impaired visual cortical plasticity both within and across days. At 8 months of age, visual plasticity is reduced in Tau- animals, and practically abolished in Tau+ animals, potentially indicating a combined effect of age and tauopathy. In addition, we found an absence of behavioural responses to novel visual stimuli in Tau+ animals. Overall, these data indicate that tauassociated neurodegeneration has an impact on both long and short-term visual plasticity, and their potential behavioural correlates.

\section{Visual cortical plasticity in neurodegeneration}

We have shown that naive visual evoked responses in V1 of rTg4510 mice are largely unaffected in Tau+ animals, even at advanced stages of neurodegeneration (8 months). These observations are consistent with previous work which shows robust orientation and direction selectivity in V1 of APP and rTg4510 mouse models $^{7-9}$. The limited impact of degeneration on these basic visual properties of cortical neurons may suggest that the accumulation of tau is not sufficient to disrupt neuronal function ${ }^{9}$. However, orientation and direction selectivity may rely primarily on the functional properties of thalamocortical relay cells, and the pattern of their cortical projections, rather than intracortical operations ${ }^{24}$. These basic functional properties may therefore be resilient, because the thalamus is largely unaffected in many models of neurodegeneration, including the rTg4510 model. Functional properties that depend on cortical cellular mechanisms, and the 
balance of intra-cortical excitation and inhibition are more likely to be affected earlier in neurodegeneration 25. Our observations support this hypothesis because visual cortical plasticity, which is likely to be more dependent on these processes, is disrupted in the rTg4510 mice.

299 We monitored visual plasticity at short- and long timescales. Stimulus response potentiation (SRP) is a form of long-term plasticity that is dependent on parvalbumin-positive interneuron activity ${ }^{26}$, and is thought to coopt similar pathways to thalamocortical LTP27, including synaptic plasticity, NMDA receptor activation and increased AMPA receptor trafficking ${ }^{16,18}$. Some of these circuits, particularly parvalbumin interneurons, are also thought to be important in ocular dominance plasticity ${ }^{28,29}$. There is some evidence that inhibitory pathways are more profoundly affected early in neurodegeneration in the $\mathrm{rTg} 4510$ model $^{30}$, so our observation of reduced SRP in Tau+ animals may be consistent with disruption of ocular dominance plasticity in the visual cortex of APP and PS1 mouse models ${ }^{31,32}$.

Our experimental design allows for measurements at multiple timescales, and we found concomitant changes at short and long time scales of plasticity. Short-term visual plasticity, usually known as adaptation, is linked to transient changes in the responsivity of synapses ${ }^{33}$ or post-synaptic mechanisms related to spike frequency adaptation ${ }^{34}$. In 8-month old Tau+ animals, we found no sign of suppressive adaptation effects, even though the amplitude of the VEP was similar to that in Tau- animals, where we saw robust adaptation effects (albeit less than in 5-month old Tau- animals). Reduced adaptation effects in older Tau+ animals may reflect disruption of adaptation mechanisms in excitatory synapses or spike-frequency adaptation. There is also some evidence that adaptation effects include increased inhibition ${ }^{35}$, so absence of adaptation effects in older Tau+ animals may also reflect substantial disruption to inhibitory circuits. In 5-month old animals, adaptation effects in early blocks each day were similar in Tau+ and Tau- animals. In later blocks, adaptation effects were reduced in Tau+ animals. This reduced adaptation might reflect partial disruption to the same mechanisms that are then grossly impaired at 8-months, such that disruption to them is only revealed after prolonged bouts of stimulation. The reduced adaptation effects seen in later blocks may, however, also arise if additional suppressive adaptation effects in Tau+ animals are recruited over longer time-scales (that may exert an effect across blocks), or if mechanisms that allow recovery from the preceding block are impaired. Our results therefore suggest that even early stages of neurodegeneration have an impact on both synaptic mechanisms and intracellular trafficking in V1. We note, however, that because we measured the local field potential, our measurements reflect the pooled signal of excitatory and inhibitory synapses ${ }^{36,37}$. Spiking activity in individual neurons, which depends on idiosyncratic and finely balanced excitation and inhibition, may show variable effects.

We have shown that a simple visual paradigm allows easy characterisation of the impact of neurodegeneration on intrinsic plasticity in awake animals, both at short (seconds and minutes) and longer (days) time scales. We have also shown that plasticity over longer time scales is markedly reduced in older animals, consistent with age-related reductions in ocular dominance plasticity in mice that have been documented previously 38,39 . In humans, repetitive visual stimulation produces a lasting enhancement of VEPs as measured by EEG ${ }^{40,41}$, similar to SRP. This potentiation has been shown to be impaired in disorders that are thought to be associated with hypofunction of the NMDA receptor, like schizophrenia ${ }^{42}$. AD patients also show a deficit in NMDAR-dependent forms of cortical plasticity ${ }^{43}$. Assessing whether VEP potentiation is impaired in individuals with or at risk of $A D$, or in normal ageing, could be a subject for future studies. If true, it could provide a framework to assess disease progression in these patients that could be less invasive and cheaper than other biomarkers. Similarly, short-term visual adaptation effects are easy to measure in humans, both perceptually and via non-invasive EEG or fMRI measurements ${ }^{44}$.

Visual deficits are common in dementia, and are particularly acute in patients with posterior cortical atrophy (PCA), which affects many sufferers of Alzheimer's disease (AD ${ }^{45}$. The neural bases of these effects in PCA patients is thought to be tau-related pathological changes in posterior cortices such as the occipital lobe ${ }^{46-48}$. Recent large-scale characterisations of tau-patterning in AD show that a posterior, occipitotemporal subtype is as frequent as other subtypes ${ }^{49}$. Functional measurements of visual cortical plasticity may therefore be particularly relevant to these individuals. 


\section{Plasticity in rTg4510 mouse model of tauopathy}

346 Previous work in rTg4510 mice has shown a link between neurodegeneration and plasticity in high-level cortical circuits. rTg4510 mice show impaired long-term depression in perirhinal cortex synapses, which may underlie defects in long-term recognition memory ${ }^{50}$. Impairments in LTP are also observed in the hippocampus ${ }^{51-53}$ and frontal cortex ${ }^{54}$ of $\mathrm{rTg} 4510$ (and APP/PS1) mice, including changes in intrinsic membrane properties, depolarisation of the resting potential, increased excitability and changes in spiking dynamics. These cellular changes are accompanied by disturbed oscillations in the LFP and disordering of 'place cells' in hippocampus ${ }^{55-57}$. These deficits in both short- and medium-term neuronal plasticity are not simply a result of accumulation of insoluble NFT, and arise before pronounced changes in cellular morphology $y^{54,58,59}$. Our findings complement and extend these studies by showing that intrinsic LTP-like visual plasticity is also disrupted in rTg4510 animals in vivo, even at early stages of neurodegeneration. We have further shown that visual plasticity decreases with age even in Tau- and WT animals. Our results suggest that age should be taken into account when studying the impact of neurodegeneration on LTP-like effects in older animals. Finally, we have shown that there are substantial changes in plasticity in primary visual cortex, an area which contributes to functional responses in higher-order brain areas like the hippocampus, perirhinal and prefrontal cortices. The contribution of primary sensory cortices may therefore need to be considered when studying the impact of neurodegeneration on plasticity in higher-order areas in vivo.

The functional effects observed at early stages of neurodegeneration in rTg4510 mice may be explained by increased synaptic instability as a result of synaptic density reduction and increased dendritic spine turnover ${ }^{60}$ or may reflect mislocalization of soluble tau to dendrites ${ }^{61}$. Synaptic instability is likely to impair long-term plasticity processes and might explain the reduction in SRP that we see in Tau+ mice. Our findings of impaired short-term adaptation effects in 5-month old Tau+ mice may reflect postsynaptic abnormalities as a result of tau accumulation in dendritic spines, or a reduction in availability of excitatory synapses, which is then exacerbated in older animals. Future studies could utilise our findings in the visual cortex to study the relationship between plasticity impairments and synaptic dysfunction in neurodegeneration in vivo. The changes in short-term adaptation effects that we see in Tau+ mice provide a particularly attractive target for experimental measurements.

We note that retinal and optic nerve atrophy has been reported in late-stages (ca. 7.5 months) of rTg4510 neurodegeneration ${ }^{62}$. These changes in retinal structure may be expected to reduce the visual cortical response as measured by the VEP. However, we found that VEP amplitude was robust, even in 8-months old Tau+ animals. Tau+ mice also have altered circadian rhythms, with increased periods of wakefulness, less time in non-rapid-eye-movement sleep ${ }^{63}$, and altered sharp-wave ripple dynamics ${ }^{57}$. These changes may contribute to the changes in long-term visual plasticity that we observed as sleep is important for SRP in mouse $\mathrm{V} 117,27,64,65$.

Recent work ${ }^{66}$ shows that in the $\mathrm{rTg} 4510$ mouse model, a fragment of the Fgf14 gene was replaced by the insertion of the P301L transgene (tau- $\mathrm{Tg}$ ). This genetic disruption may influence the progress of neuronal loss and behavioural abnormalities alone or in combination with the expression of the mutant tau. Our experiments mitigate these potential offsite effects by comparing Tau- and Tau+ mice, that differ in mutant tau expression but not genotype. In addition, our comparisons of Tau- with WT littermates reveal similar longterm plasticity and visual evoked behaviour. Interestingly, older $(8 \mathrm{~m})$ Tau- mice showed reduced adaptation, or short-term plasticity but WT animals did not. Adaptation effects are generally thought to be preserved with age in humans ${ }^{67,68}$. It is possible that reduced adaptation in Tau- animals could be an effect of the Fgf14 deletion or it could be an effect of tau accumulation before the onset of the doxycycline treatment (which started at 2 months). If the latter is true, this would render adaptation as a more sensitive assay to assess functional changes in neurodegeneration in older animals.

Previous studies have reported alterations in rTg4510 mouse behaviour: Tau+, and to a lesser extent, Taumice tend to show hyperactivity and motor stereotypy, whilst maintaining good motor coordination 19,21,22,63,69. These mice do not appear to respond to novelty, and have impaired spatial working memory ${ }^{19,21}$. In our experiments on head-fixed animals, Tau+ animals reacted less to the appearance of a new visual stimulus than did their Tau- or WT counterparts, consistent with reduced novelty responses. This insensitivity to novelty may partly reflect a lack of arousal or a deficit in attention, which may in turn indicate deficiencies in 
noradrenaline circuits that appear critical for these processes ${ }^{70}$. The CamKIla promoter that drives transgene expression in the rTg4510 mouse is likely to be expressed in locus coeruleus ${ }^{71}$, and it is therefore possible that the noradrenalin input to cortex is disrupted in Tau+ mice.

\section{Conclusions}

401 Plasticity is a hallmark of neuronal function, important for learning and memory. Neurodegenerative diseases like $A D$ not only disrupt neuronal structure and function, but erode the flexibility of neurons and circuits. Failure of synaptic plasticity is assumed to occur early in the course of AD. We verified the effect of tauopathy on an intrinsic form of sensory learning and memory in $\mathrm{V} 1$ of mouse. We found impaired visual plasticity even at early stages of neurodegeneration, before substantial neuronal loss occurs. Our measurements offer a simple and direct readout of intrinsic plasticity in degenerating brain circuits, and a potential target for understanding, detecting and tracking that neurodegeneration, in humans as well as in animal models.

\section{Methods}

\section{Animal Experiments}

410 All experiments were performed in accordance with the Animals (Scientific Procedures) Act 1986 (United 411 Kingdom) and Home Office (United Kingdom) approved project and personal licenses. The experiments were 412 approved by the University College London Animal Welfare Ethical Review Board under Project License $41370 / 8637$.

414 The generation of $\mathrm{rTg} 4510$ transgenic mice was performed as described previously ${ }^{11,19}$. A total of 50 male transgenic mice and 16 wild-type (WT) littermates were obtained at approximately 7 weeks of age from Eli Lilly and Company (Windlesham, UK) via Envigo (Loughborough, UK). At 8 weeks of age, to suppress tau expression, 25 of the 50 transgenic mice were treated with Doxycycline, which included four $10 \mathrm{mg} / \mathrm{kg}$ bolus oral doses of doxycycline (Sigma) in $5 \%$ glucose solution by oral gavage across 4 days, followed by ad libitum access to Teklad base diet containing 200ppm doxycycline (Envigo) for the duration of the experiment. The mice in this group were designated 'Tau-' animals. The remaining 25 animals, designated as 'Tau+', and the WT animals received 4 oral doses of the vehicle ( $5 \%$ glucose) and had ad libitum access to standard feed for the duration of the experiment. All animals also had ad libitum access to water. Mice were subsequently divided into two cohorts. One cohort was tested at approximately 5 months (22-26 weeks, 12 Tau-, 12 Tau+, $6 \mathrm{WT}$ ), and another cohort was tested at approximately 8 months of age (31-35 weeks, 13 Tau-, 13 Tau+, $10 \mathrm{WT}$ ). Mice were group housed to a maximum of 5 individuals per cage until 3 days before surgery, when they were separated into individual cages. All animals were kept under a 12-hour light/dark cycle, and both behavioural and electrophysiological recordings were carried out during the dark phase of the cycle.

\section{Surgery}

429 Mice were anaesthetised for surgery with $3 \%$ isoflurane in $\mathrm{O}_{2}$. Preoperative analgesia (Carprieve, $5 \mathrm{mg} / \mathrm{kg}$ ) was given subcutaneously and lubricant ophthalmic ointment was applied. Anaesthesia was maintained with $1-2 \%$ isoflurane in $\mathrm{O}_{2}$ and the depth was monitored by absence of pinch-withdrawal reflex and breathing rate. The body temperature was maintained using a heating blanket. A small craniotomy hole $\left(<1 \mathrm{~mm}^{2}\right)$ was made over the right primary visual cortex $(2.8 \mathrm{~mm}$ lateral and $0.5 \mathrm{~mm}$ anterior from lambda) and a chronic local field potential (LFP) recording electrode (Bear lab chronic microelectrode Monopolar 30070, FHC, USA) was implanted approximately $400-450 \mu \mathrm{m}$ below the cortical surface. A ground screw was implanted over the left prefrontal cortex and a custom-built stainless-steel metal plate was affixed on the skull. Dental cement (Super-Bond C\&B, Sun Medical) was used to cover the skull, ground screw and metal plate, enclosing and stabilizing the electrode. Analgesic treatment (Metacam, Boehringer Ingelheim, 1mg/kg) mixed in condensed milk was provided orally for three days after the surgery. Mice recovered for at least 7 days before the first recording session.

\section{Visual Stimulus Presentation \& Experimental Setup}

442 Visual stimuli consisted of full-field, 100\% contrast sinusoidal gratings generated using BonVision ${ }^{72}$, presented on a y-corrected computer monitor (liyama ProLite EE1890SD). The gratings were presented in a circular aperture with hard edges, outside of which the monitor was held at the mean luminance ('grey screen'). The grating was oriented at either $-45^{\circ}$ or $45^{\circ}$ from vertical, and reversed contrast (flickered) at a 
446 frequency of $1.95 \mathrm{~Hz}$. The display was placed $15 \mathrm{~cm}$ from - and normal to - the mouse and centred on the 447 left visual field. The stimulus was warped to maintain visual angle across the monitor.

448 One week after the surgery, mice were placed on a styrofoam wheel with a grey screen and habituated over mins. Mice were allowed to run on the wheel, and their speed was recorded using a rotary encoder. Pupils were imaged using an infrared camera camera (DMK 22BUC03, ImagingSource; $30 \mathrm{~Hz}$ ) focused on the left eye through a zoom lens (Computar MLH-10X Macro Zoom Lens), and acquired by the same computer that presented the visual stimulus. Pupil estimates (position, diameter) were tracked online using custom routines in Bonsai. At the beginning of each recording session, mice were presented with a grey screen for 3-5min. On the first day of recordings, mice were presented with 5 blocks of a grating oriented at $45^{\circ}$ and 5 blocks of a grating oriented at $-45^{\circ}$, alternating between the two. Each block consisted of 200 continuous reversals, and were separated by $30 \mathrm{~s}$, during which the monitor was held at the mean luminance. For 6 animals ( $2 \mathrm{WT}$, 2 Tau- and $2 \mathrm{Tau}+$ ), each block consisted of 400 phase reversals. On days 2-8, mice were presented with 10 blocks of a single oriented grating (familiar stimulus), that was either $45^{\circ}$ or $-45^{\circ}$, randomly assigned for each animal (counterbalanced between groups). The last day of recordings, day 9 , was similar to day 1 . For days 1 and 9, whether the first block presented would be the familiar or the unfamiliar stimulus (presented only on the first and last day of recordings), was randomly assigned for each animal.

\section{Neural Recordings}

464 Signals from the recording electrode were acquired, digitised and filtered using an OpenEphys acquisition

board connected to a different computer from that used to generate the visual stimulus. The electrophysiological and rotary wheel signals were sampled at $30 \mathrm{kHz}$. These data were synchronised with pupil video recordings and visual stimulus via the signal of a photodiode (PDA25K2, Thorlabs, Inc., USA) that monitored timing pulses on a small corner of the monitor shielded from the animal.

\section{Data analysis}

470 All data were analysed using custom software written in MATLAB (MathWorks). Neural and wheel data were 471 filtered with an 8th order Chebyshev Type I lowpass filter and downsampled to $1 \mathrm{kHz}$.

472 VEP analysis: VEPs were averaged across all phase reversals and blocks for each stimulus on each day. 473 To estimate the VEP amplitude, the LFP signal was filtered using a second order bandpass filter with a $0.3 \mathrm{~Hz}$ 474 low cut and $50 \mathrm{~Hz}$ high cut frequency. The negative trough was defined as the minimum value within the first $475150 \mathrm{~ms}$ after stimulus reversal, and the positive peak was defined as the maximum value within the first $476250 \mathrm{~ms}$. The amplitude was defined as the difference between this trough and peak.

477 Pupil data: Eye blinks were removed by identifying any points that were two times above the variance of the 478 eye position. Removed values were replaced using nearest neighbour interpolation. Pupil area (in pixels) was converted to $\mathrm{mm}^{2}$ by multiplying with the square of the camera resolution (in $\mathrm{mm} / \mathrm{pixel}$ ). Responses were then normalised to the average pupil area in the two minutes before the stimulus onset, where animals were viewing a grey screen.

Wheel data: We estimated the speed and direction of the rotating wheel using a quadrature encoder. Rotations were converted to speed by multiplying with the wheel circumference and dividing by the encoder resolution. Speed was then smoothed using a gaussian filter with a $50 \mathrm{~ms}$ window.

Regression: We fitted an elastic net regularization model to predict the VEP amplitude for each animal using days, block number within each day, movement speed and pupil area as regressors. Regressors and predicted value were normalized to range between 0 and 1 before fitting. We explored the entire regularisation path by using $N$ values $(<=10,000)$ of the regularisation hyperparameter, fixing the ratio of the $\lambda 1$ and $\lambda 2$-norm regularisers to 2 . The impact $M$ of each regressor $r$ in predicting the VEP amplitude was calculated as:

$$
M\left(r_{i}\right)=\sum_{l=1}^{L} \sum_{t=1}^{T} w_{i, l} r_{i, t} / \sum_{l=1}^{L} \sum_{t=1}^{T} \hat{y}_{l, t}
$$


Where $l$ is the regularization hyperparameter, $t$ denotes each time there is a stimulus reversal $(t=$ 1 : number of reversal each day $*$ number of days), $w$ is the learned weight, $r_{i}$ denotes each respective regressor (where $i$ is either days, block number, speed, pupil area) and $y$ is the predicted value of the elastic net model. Note that if two or more regressors are correlated, the regularization model can assign weights to one or both regressors.

Visual adaptation: To estimate the adaptation effect for each animal, for each day, the VEP amplitude was calculated within a block using a step of 20 reversals. The mean amplitude of the last 200 reversals was subtracted from each block and amplitudes were averaged over blocks 2-10, or blocks 2-5, or blocks 6-10. A decaying exponential function was fitted to the averaged data using least squares. The exponential time constant for each animal was fixed to $\tau=8.4$ reversals. This value was calculated by fitting the exponential to the mean trace obtained by averaging over all animals, for days 2-8, and blocks 2-10.

501

502

503

504

505

506

507

508

509

510

511

512

513

514

515

516

517

518

519

520

521

522

523

524

525

526

527

528

529

530

\section{Acknowledgements}

\section{Brain samples}

\section{Histopathology} in a blinded fashion.

\section{Statistical analyses}

Mice were euthanised by overdose injection of pentobarbital (intraperitoneal) and perfused with phosphatebuffered saline (PBS). Brains were removed, weighed, and the right hemisphere was fixed in $10 \%$ buffered formalin until processed (7-13 months) for immunohistochemistry pathology assessment.

Immunohistochemistry was performed for all mice. The brains were immersed in PBS that contained 30\% sucrose, and subsequently cut into $40 \mu \mathrm{m}$ parasagittal sections on a cryostat (Leica CM1520). Antigen retrieval was achieved through heating sections in citrate buffer ( $\mathrm{pH} 6.0$; Vector Labs) in an oven at $60^{\circ} \mathrm{C}$ overnight. Slides were treated with $0.3 \%$ hydrogen peroxide solution ( $3 \%$ in distilled water) for $10 \mathrm{~min}$ to eliminate endogenous peroxidase activity, and subsequently washed with PBS with $0.5 \%$ Triton X-100. Immunohistochemistry was performed using a primary antibody for tau phosphorylated at serine 202 (mouse monoclonal AT8, 1:1000, Thermo Fisher Scientific). The Mouse on Mouse (MOM) Detection Kit (Vector Labs, BMK-2202) was used for staining, with buffers prepared as described in standard protocol supplied with the kit. After rinsing, slides were treated for 5 min with the chromogen 3,3'-diaminobenzidine (DAB; Vector Laboratories, SK-4105) to allow visualisation. The slides were then coverslipped with Shandon ClearVue Mountant XYL (Thermofisher) and digitised using a Leica Microscope (DMi8 S) coupled with a Leica camera (DFC7000 GT; Leica Microsystems). The Fiji image processing package was used to view the digitized tissue sections ${ }^{73}$. At least 3 regions of interest (ROIs) of the same size were selected from approximately the same brain location in V1 and hippocampus for each animal. In cases where V1 sections were fragmented $(n=12)$, additional ROls were selected from elsewhere in the cortex. To assess the tau burden, a normal distribution was fit to the image of each ROI and the mean and standard deviation were obtained and averaged over all ROIs for each animal (Fig. S1). To compare the tau burden in V1 and hippocampus, the mean values obtained from the fitted distributions were zscored and sign inverted (Fig. 1). These analyses were performed

All data are presented as a mean \pm standard error of the mean (SEM). Statistical comparisons were performed in MATLAB and SPSS (IBM). A two-way or a repeated-measures ANOVA was applied for all comparisons. $p<0.05$ was used as the significance threshold. Exact $p$ values are given.

We thank Francesca Cacucci (F. C.) for comments on the manuscript. We thank Zeshan Ahmed, and Anthony Blockeel for useful advice and discussions. This work was supported by the Medical Research Council grant (R023808) to S.G.S., A.B.S. and F.C., a Biotechnology and Biological Sciences Research Council grant (R004765) to S.G.S. and A.B.S., a Sir Henry Dale Fellowship from the Wellcome Trust and Royal Society (200501) to A.B.S, and an International Collaboration Award to S.G.S (with Adam Kohn) from the Stavros Niarchos Foundation / Research to Prevent Blindness. 


\section{Author Contributions}

539 Conceptualization, A.P., A.B.S., and S.G.S.; Methodology, A.P., F.R.R., A.B.S., and S.G.S.; Investigation,

540 A.P., F.R.R., and J.H.; Validation, Formal Analysis, Data Curation, A.P., F.R.R., S.G.S.; Writing - Original 541 Draft, A.P. and F.R.R.; Writing - Review \& Editing, A.P., F.R.R., A.B.S., and S.G.S.; Visualization, A.P., 542 F.R.R., A.B.S., and S.G.S.; Funding Acquisition, A.B.S., and S.G.S.; Resources, A.B.S., K.P. and S.G.S.; 543 Supervision, A.B.S., and S.G.S.

\section{References}

$1 \quad$ Chapman, P. F. et al. Impaired synaptic plasticity and learning in aged amyloid precursor protein transgenic mice. Nat Neurosci 2, 271-276, doi:10.1038/6374 (1999).

2 Shipton, O. A. et al. Tau protein is required for amyloid \{beta\}-induced impairment of hippocampal long-term potentiation. J Neurosci 31, 1688-1692, doi:10.1523/JNEUROSCI.2610-10.2011 (2011). Spires-Jones, T. \& Knafo, S. Spines, plasticity, and cognition in Alzheimer's model mice. Neural Plast 2012, 319836, doi:10.1155/2012/319836 (2012).

4 Biundo, F., Del Prete, D., Zhang, H., Arancio, O. \& D'Adamio, L. A role for tau in learning, memory and synaptic plasticity. Sci Rep 8, 3184, doi:10.1038/s41598-018-21596-3 (2018).

$5 \quad$ Klyubin, l. et al. Amyloid beta protein immunotherapy neutralizes Abeta oligomers that disrupt synaptic plasticity in vivo. Nat Med 11, 556-561, doi:10.1038/nm1234 (2005).

6 Walsh, D. M. et al. Naturally secreted oligomers of amyloid beta protein potently inhibit hippocampal long-term potentiation in vivo. Nature 416, 535-539, doi:10.1038/416535a (2002).

$7 \quad$ Grienberger, C. et al. Staged decline of neuronal function in vivo in an animal model of Alzheimer's disease. Nat Commun 3, 774, doi:10.1038/ncomms1783 (2012).

8 Liebscher, S., Keller, G. B., Goltstein, P. M., Bonhoeffer, T. \& Hubener, M. Selective Persistence of Sensorimotor Mismatch Signals in Visual Cortex of Behaving Alzheimer's Disease Mice. Curr Biol 26, 956-964, doi:10.1016/j.cub.2016.01.070 (2016). Kuchibhotla, K. V. et al. Neurofibrillary tangle-bearing neurons are functionally integrated in cortical circuits in vivo. Proc Natl Acad Sci U S A 111, 510-514, doi:10.1073/pnas.1318807111 (2014). Ramsden, M. et al. Age-dependent neurofibrillary tangle formation, neuron loss, and memory impairment in a mouse model of human tauopathy (P301L). J Neurosci 25, 10637-10647, doi:10.1523/JNEUROSCI.3279-05.2005 (2005).

Santacruz, K. et al. Tau suppression in a neurodegenerative mouse model improves memory function. Science 309, 476-481, doi:10.1126/science.1113694 (2005). Yue, M., Hanna, A., Wilson, J., Roder, H. \& Janus, C. Sex difference in pathology and memory decline in rTg4510 mouse model of tauopathy. Neurobiol Aging 32, 590-603, doi:10.1016/j.neurobiolaging.2009.04.006 (2011).

Spires, T. L. et al. Region-specific dissociation of neuronal loss and neurofibrillary pathology in a mouse model of tauopathy. Am J Pathol 168, 1598-1607, doi:10.2353/ajpath.2006.050840 (2006). Solomon, S. G. \& Kohn, A. Moving sensory adaptation beyond suppressive effects in single neurons. Curr Biol 24, R1012-1022, doi:10.1016/j.cub.2014.09.001 (2014). Webster, M. A. Visual Adaptation. Annu Rev Vis Sci 1, 547-567, doi:10.1146/annurev-vision082114-035509 (2015). Frenkel, M. Y. et al. Instructive effect of visual experience in mouse visual cortex. Neuron 51, 339349, doi:10.1016/j.neuron.2006.06.026 (2006).

Aton, S. J., Suresh, A., Broussard, C. \& Frank, M. G. Sleep promotes cortical response potentiation following visual experience. Sleep 37, 1163-1170, doi:10.5665/sleep.3830 (2014). Cooke, S. F., Komorowski, R. W., Kaplan, E. S., Gavornik, J. P. \& Bear, M. F. Visual recognition memory, manifested as long-term habituation, requires synaptic plasticity in V1. Nat Neurosci 18, 262-271, doi:10.1038/nn.3920 (2015). Blackmore, T. et al. Tracking progressive pathological and functional decline in the rTg4510 mouse model of tauopathy. Alzheimers Res Ther 9, 77, doi:10.1186/s13195-017-0306-2 (2017). Niell, C. M. \& Stryker, M. P. Modulation of visual responses by behavioral state in mouse visual cortex. Neuron 65, 472-479, doi:10.1016/j.neuron.2010.01.033 (2010). Wes, P. D. et al. Tau overexpression impacts a neuroinflammation gene expression network perturbed in Alzheimer's disease. PLoS One 9, e106050, doi:10.1371/journal.pone.0106050 (2014). Cook, C. et al. Severe amygdala dysfunction in a MAPT transgenic mouse model of frontotemporal dementia. Neurobiol Aging 35, 1769-1777, doi:10.1016/j.neurobiolaging.2013.12.023 (2014). 
Helboe, L., Egebjerg, J., Barkholt, P. \& Volbracht, C. Early depletion of CA1 neurons and late neurodegeneration in a mouse tauopathy model. Brain Res 1665, 22-35,

doi:10.1016/j.brainres.2017.04.002 (2017).

Priebe, N. J. Mechanisms of Orientation Selectivity in the Primary Visual Cortex. Annu Rev Vis Sci 2, 85-107, doi:10.1146/annurev-vision-111815-114456 (2016).

Rubin, D. B., Van Hooser, S. D. \& Miller, K. D. The stabilized supralinear network: a unifying circuit motif underlying multi-input integration in sensory cortex. Neuron $\mathbf{8 5}, 402-417$,

doi:10.1016/j.neuron.2014.12.026 (2015).

Kaplan, E. S. et al. Contrasting roles for parvalbumin-expressing inhibitory neurons in two forms of adult visual cortical plasticity. Elife 5, doi:10.7554/eLife.11450 (2016).

Cooke, S. F. \& Bear, M. F. Visual experience induces long-term potentiation in the primary visual cortex. J Neurosci 30, 16304-16313, doi:10.1523/JNEUROSCI.4333-10.2010 (2010).

Hensch, T. K. Critical period plasticity in local cortical circuits. Nat Rev Neurosci 6, 877-888, doi:10.1038/nrn1787 (2005).

Hooks, B. M. \& Chen, C. Circuitry Underlying Experience-Dependent Plasticity in the Mouse Visual System. Neuron 107, 986-987, doi:10.1016/j.neuron.2020.08.004 (2020).

Shimojo, M. et al. Selective Disruption of Inhibitory Synapses Leading to Neuronal Hyperexcitability at an Early Stage of Tau Pathogenesis in a Mouse Model. J Neurosci 40, 3491-3501,

doi:10.1523/JNEUROSCI.2880-19.2020 (2020).

William, C. M. et al. Synaptic plasticity defect following visual deprivation in Alzheimer's disease model transgenic mice. J Neurosci 32, 8004-8011, doi:10.1523/JNEUROSCI.5369-11.2012 (2012). Maya-Vetencourt, J. F., Carucci, N. M., Capsoni, S. \& Cattaneo, A. Amyloid plaque-independent deficit of early postnatal visual cortical plasticity in the 5XFAD transgenic model of Alzheimer's disease. J Alzheimers Dis 42, 103-107, doi:10.3233/JAD-140453 (2014).

Regehr, W. G. Short-term presynaptic plasticity. Cold Spring Harb Perspect Biol 4, a005702, doi:10.1101/cshperspect.a005702 (2012).

Sanchez-Vives, M. V., Nowak, L. G. \& McCormick, D. A. Cellular mechanisms of long-lasting adaptation in visual cortical neurons in vitro. J Neurosci 20, 4286-4299 (2000).

Heintz, T. G., Hinojosa, A. J. \& Lagnado, L. Opposing forms of adaptation in mouse visual cortex are controlled by distinct inhibitory microcircuits and gated by locomotion. bioRxiv,

2020.2001.2016.909788, doi:10.1101/2020.01.16.909788 (2020).

Einevoll, G. T., Kayser, C., Logothetis, N. K. \& Panzeri, S. Modelling and analysis of local field potentials for studying the function of cortical circuits. Nat Rev Neurosci 14, 770-785,

doi:10.1038/nrn3599 (2013).

Kim, T., Chaloner, F. A., Cooke, S. F., Harnett, M. T. \& Bear, M. F. Opposing Somatic and Dendritic Expression of Stimulus-Selective Response Plasticity in Mouse Primary Visual Cortex. Front Cell Neurosci 13, 555, doi:10.3389/fncel.2019.00555 (2019).

Lehmann, K. \& Lowel, S. Age-dependent ocular dominance plasticity in adult mice. PLoS One 3, e3120, doi:10.1371/journal.pone.0003120 (2008).

Lehmann, K., Schmidt, K. F. \& Lowel, S. Vision and visual plasticity in ageing mice. Restor Neurol Neurosci 30, 161-178, doi:10.3233/RNN-2012-110192 (2012). Teyler, T. J. et al. Long-term potentiation of human visual evo to humans: a novel method for noninvasive assessment of cortical plasticity. Biol Psychiatry 71, 496-502, doi:10.1016/j.biopsych.2011.08.021 (2012). doi:10.1016/j.biopsych.2012.01.013 (2012). Battaglia, F. et al. Cortical plasticity in Alzheimer's disease in humans and rodents. Biol Psychiatry 62, 1405-1412, doi:10.1016/j.biopsych.2007.02.027 (2007). doi:10.1016/j.cortex.2015.10.026 (2016).

Crutch, S. J., Yong, K. X. \& Shakespeare, T. J. Looking but not seeing: Recent perspectives on posterior cortical atrophy. Current Directions in Psychological Science 25, 251-260 (2016).

Hof, P. R., Bouras, C., Constantinidis, J. \& Morrison, J. H. Selective disconnection of specific visual association pathways in cases of Alzheimer's disease presenting with Balint's syndrome. $J$ Neuropathol Exp Neurol 49, 168-184, doi:10.1097/00005072-199003000-00008 (1990). atrophy. Neurology 63, 1168-1174, doi:10.1212/01.wnl.0000140289.18472.15 (2004). 
Ossenkoppele, R. et al. Tau, amyloid, and hypometabolism in a patient with posterior cortical atrophy. Ann Neurol 77, 338-342, doi:10.1002/ana.24321 (2015).

Vogel, J. W. et al. Four distinct trajectories of tau deposition identified in Alzheimer's disease. Nat Med, doi:10.1038/s41591-021-01309-6 (2021).

Scullion, S. E., Barker, G. R. I., Warburton, E. C., Randall, A. D. \& Brown, J. T. Muscarinic Receptor-Dependent Long Term Depression in the Perirhinal Cortex and Recognition Memory are Impaired in the rTg4510 Mouse Model of Tauopathy. Neurochem Res 44, 617-626, doi:10.1007/s11064-018-2487-x (2019).

Hoover, B. R. et al. Tau mislocalization to dendritic spines mediates synaptic dysfunction independently of neurodegeneration. Neuron 68, 1067-1081, doi:10.1016/j.neuron.2010.11.030 (2010).

Booth, C. A. et al. Altered Intrinsic Pyramidal Neuron Properties and Pathway-Specific Synaptic Dysfunction Underlie Aberrant Hippocampal Network Function in a Mouse Model of Tauopathy. $J$ Neurosci 36, 350-363, doi:10.1523/JNEUROSCI.2151-15.2016 (2016).

Gelman, S., Palma, J., Tombaugh, G. \& Ghavami, A. Differences in Synaptic Dysfunction Between rTg4510 and APP/PS1 Mouse Models of Alzheimer's Disease. J Alzheimers Dis 61, 195-208, doi:10.3233/JAD-170457 (2018).

Crimins, J. L., Rocher, A. B. \& Luebke, J. I. Electrophysiological changes precede morphological changes to frontal cortical pyramidal neurons in the rTg4510 mouse model of progressive tauopathy. Acta Neuropathol 124, 777-795, doi:10.1007/s00401-012-1038-9 (2012).

Cheng, J. \& Ji, D. Rigid firing sequences undermine spatial memory codes in a neurodegenerative mouse model. Elife 2, e00647, doi:10.7554/eLife.00647 (2013).

Ciupek, S. M., Cheng, J., Ali, Y. O., Lu, H. C. \& Ji, D. Progressive functional impairments of hippocampal neurons in a tauopathy mouse model. J Neurosci 35, 8118-8131, doi:10.1523/JNEUROSCI.3130-14.2015 (2015).

Witton, J. et al. Disrupted hippocampal sharp-wave ripple-associated spike dynamics in a transgenic mouse model of dementia. J Physiol 594, 4615-4630, doi:10.1113/jphysiol.2014.282889 (2016).

Rocher, A. B. et al. Structural and functional changes in tau mutant mice neurons are not linked to the presence of NFTs. Exp Neurol 223, 385-393, doi:10.1016/j.expneurol.2009.07.029 (2010). Jackson, J. S. et al. Differential aberrant structural synaptic plasticity in axons and dendrites ahead of their degeneration in tauopathy. bioRxiv, 2020.2004.2029.067629, doi:10.1101/2020.04.29.067629 (2020). Jackson, J. S. et al. Altered Synapse Stability in the Early Stages of Tauopathy. Cell Rep 18, 30633068, doi:10.1016/j.celrep.2017.03.013 (2017).

61 Zhao, X. et al. Caspase-2 cleavage of tau reversibly impairs memory. Nat Med 22, 1268-1276, doi:10.1038/nm.4199 (2016). Harrison, I. F. et al. Optic nerve thinning and neurosensory retinal degeneration in the rTg4510 mouse model of frontotemporal dementia. Acta Neuropathol Commun 7, 4, doi:10.1186/s40478018-0654-6 (2019). Holton, C. M. et al. Longitudinal changes in EEG power, sleep cycles and behaviour in a tau model of neurodegeneration. Alzheimers Res Ther 12, 84, doi:10.1186/s13195-020-00651-0 (2020). Ji, D. \& Wilson, M. A. Coordinated memory replay in the visual cortex and hippocampus during sleep. Nat Neurosci 10, 100-107, doi:10.1038/nn1825 (2007).

Cooke, S. F. \& Bear, M. F. Stimulus-selective response plasticity in the visual cortex: an assay for the assessment of pathophysiology and treatment of cognitive impairment associated with psychiatric disorders. Biol Psychiatry 71, 487-495, doi:10.1016/j.biopsych.2011.09.006 (2012). Gamache, J. et al. Factors other than hTau overexpression that contribute to tauopathy-like phenotype in rTg4510 mice. Nat Commun 10, 2479, doi:10.1038/s41467-019-10428-1 (2019). Elliott, S. L., Hardy, J. L., Webster, M. A. \& Werner, J. S. Aging and blur adaptation. J Vis 7, 8, doi:10.1167/7.6.8 (2007).

Elliott, S. L., Werner, J. S. \& Webster, M. A. Individual and age-related variation in chromatic contrast adaptation. J Vis 12, 11, doi:10.1167/12.8.11 (2012). Jul, P. et al. Hyperactivity with Agitative-Like Behavior in a Mouse Tauopathy Model. J Alzheimers Dis 49, 783-795, doi:10.3233/JAD-150292 (2016). Sara, S. J. The locus coeruleus and noradrenergic modulation of cognition. Nat Rev Neurosci 10, 211-223, doi:10.1038/nrn2573 (2009). Glennon, E. et al. Locus coeruleus activation accelerates perceptual learning. Brain Res 1709, 3949, doi:10.1016/j.brainres.2018.05.048 (2019). 
73 Schindelin, J. et al. Fiji: an open-source platform for biological-image analysis. Nat Methods 9, 676682, doi:10.1038/nmeth.2019 (2012).

\section{Supplementary Figures}
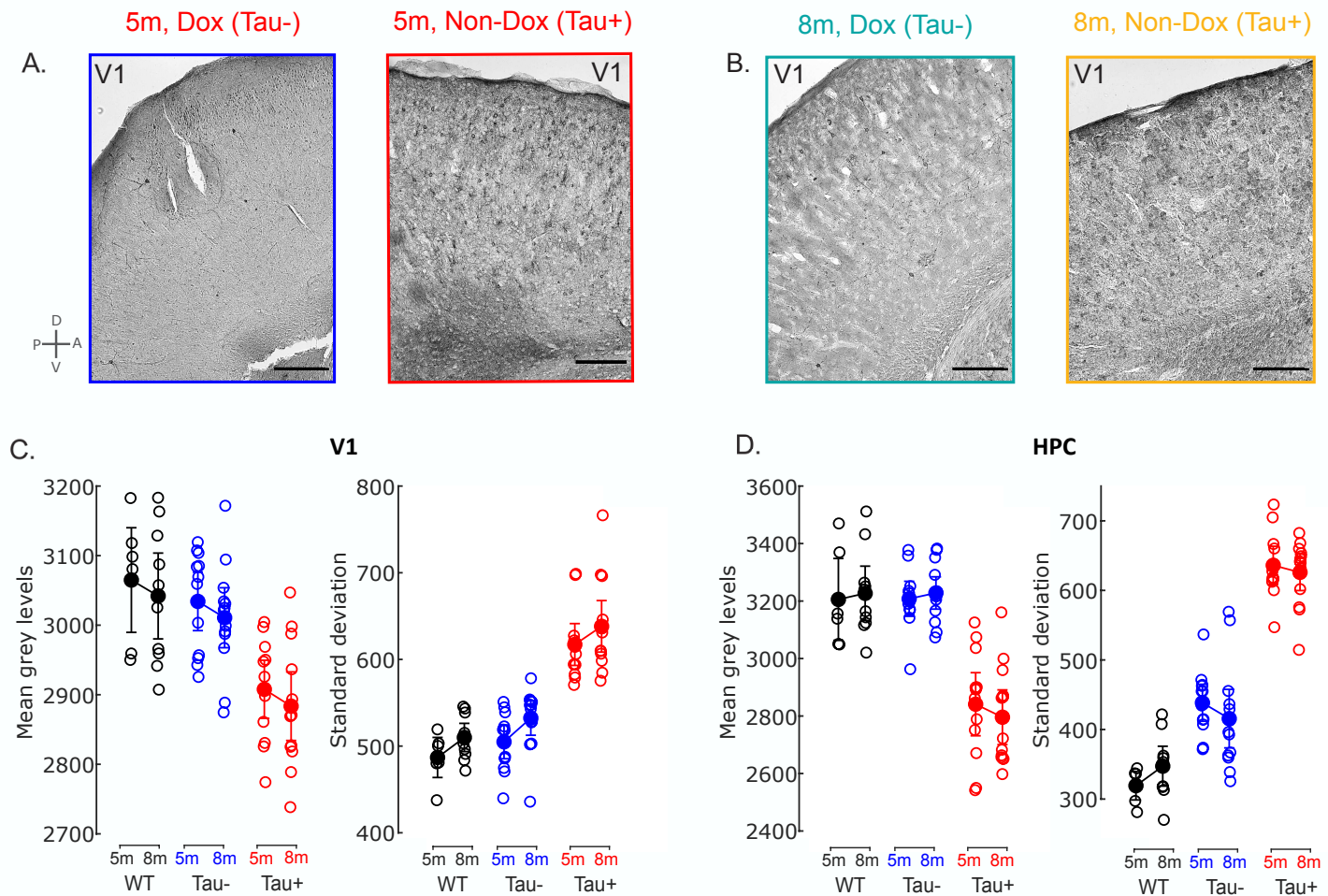

Figure S1: Immunohistochemical profiling of 5- and 8-month old rTg4510 mice. A-B. Representative immunohistochemical images from the $\mathrm{V} 1$ of Tau- mice receiving doxycycline (Dox) treatment and Tau+ mice (Non-Dox) at 5 months old (A) and 8 months old (B). C. Average of the mean grey levels (left) and standard deviation (right) of ROls selected in V1 for each animal (Methods). Data are represented as mean $\pm 2^{*}$ SEM. D. Same as in C. for hippocampal ROIs. Tau+ animals were characterised by a smaller mean and larger standard deviation compared to Tau- and WT animals, confirming that the doxycycline treatment was successful. 

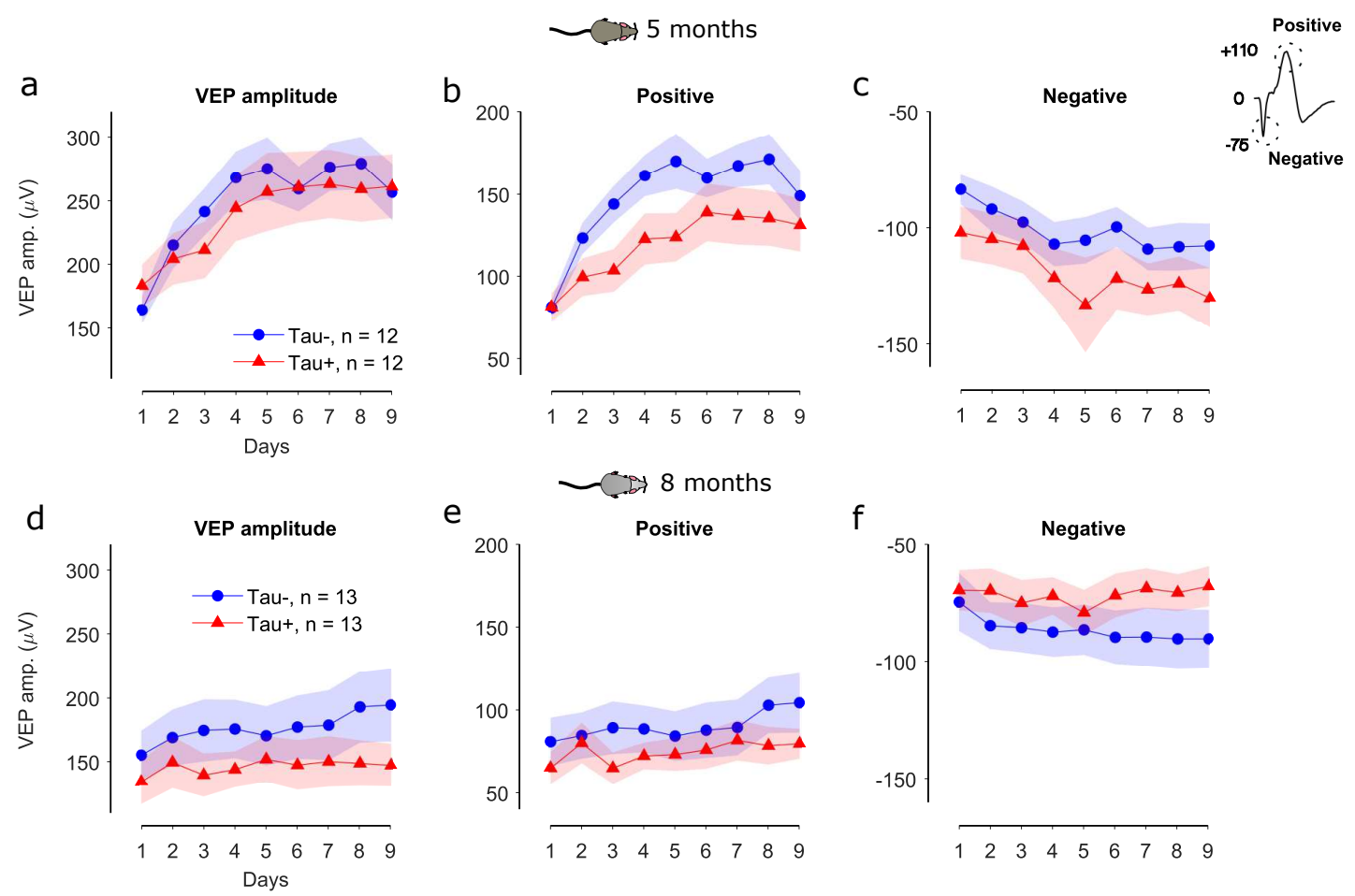

Figure S2: Differences in visual plasticity between Tau+ and Tau- animals are attributed mainly to the positive deflection of the VEP signal. a. Average VEP amplitude, defined as the difference between the positive and negative peaks of the VEP signal, across days. b. Difference between the positive deflection of the VEP signal and the baseline as a function of days, averaged across Tau+ and Tauanimals. c. Difference between the negative deflection of the VEP signal and the baseline as a function of days, averaged across the groups of animals. d-f. Same for 8-month old animals.
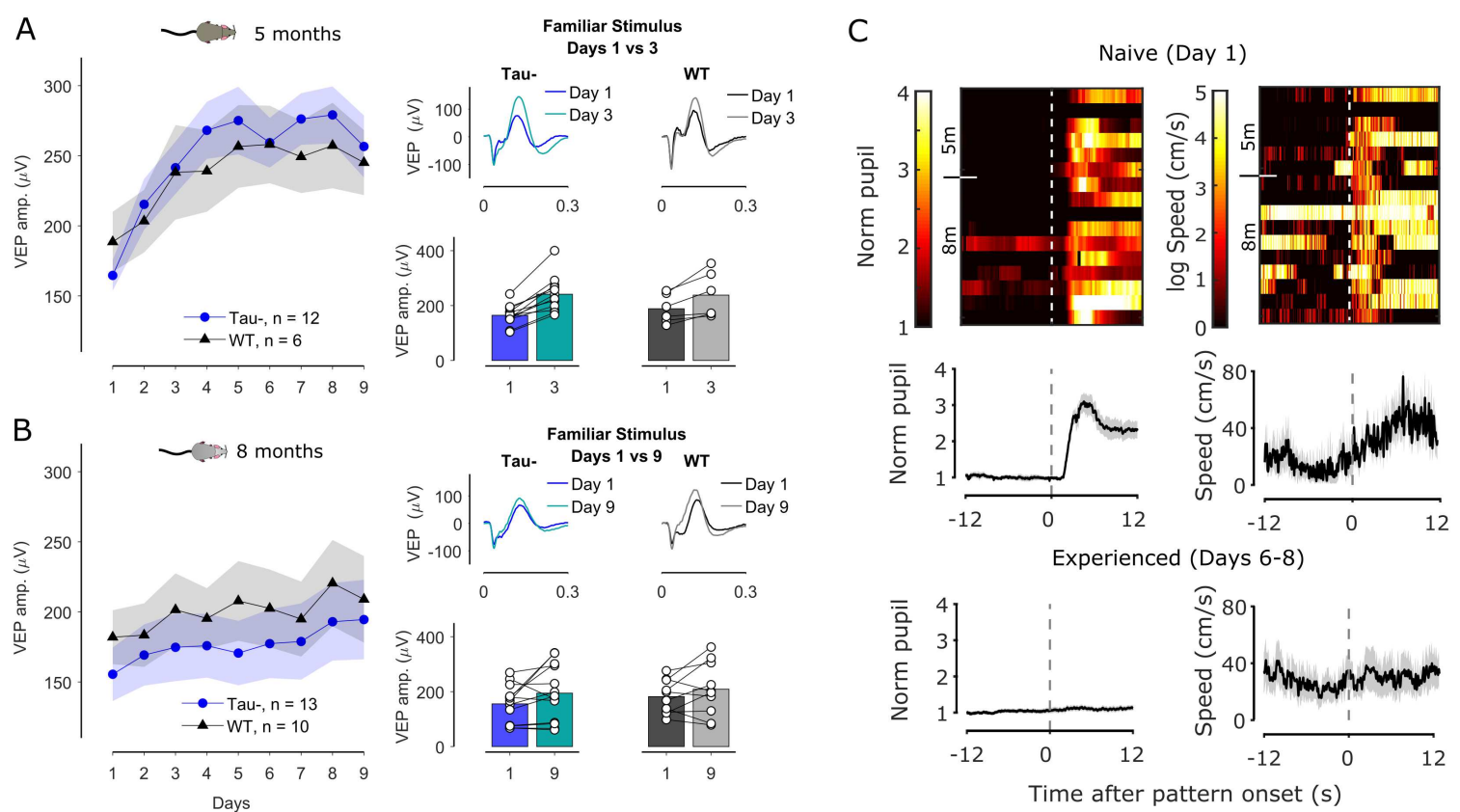

Figure S3: VEP and behavioural responses are similar in Tau- and Wild Type (WT) mice. A. Average VEP amplitude as a function of days for 5-month old Tau- mice (blue) and WT littermates (black). The panels on the right show the average VEP signal on top and the VEP amplitude of individual animals on 
the bottom for days 1 and 3 . VEPs significantly increased by day 3 relative to day 1 for the familiar stimulus for both Tau- and WT animals. B. Average VEP amplitude as a function of days for 8-month old Tau- mice (blue) and WT littermates (black). The panels on the right show the average VEP signal and the VEP amplitude of individual animals for days 1 and 9. WT mice had on average a larger VEP amplitude than Tau- mice but the VEPs increased at a similar rate as a function of days. The rate and magnitude of potentiation was reduced compared to 5-month old animals. C. Left: Images and average responses of the normalized pupil to the onset of the stimulus of the first block presented for naive (top) and experienced (bottom) WT mice. Right: Images and average responses of the movement speed.

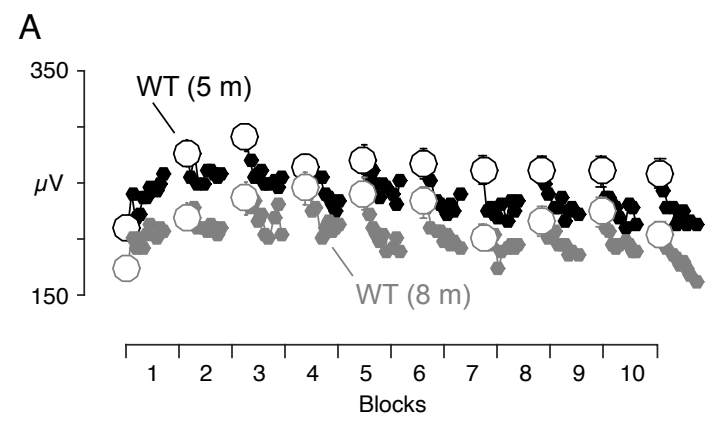

B
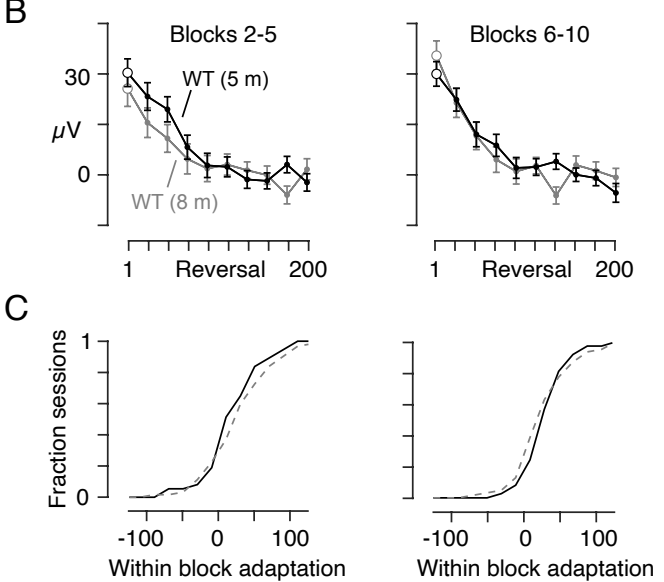

Figure S4: Adaptation in WT animals does not reduce with age (related to Figure 4). A. Average VEP amplitude as a function of block number for 5-month old WT mice (black) and 8-month old WT mice (grey). The VEP amplitude was calculated from non-overlapping averages of 20 reversals. With the notable exception of the first block, VEPs showed a reduction of responses within each block, consistent with classic sensory adaptation effects. B. Average VEP responses for blocks 2-5 (Left) and blocks 6-10 (Right) on days 2-8. C. Cumulative histograms of the fitted amplitudes showing no significant differences between 5and 8-month old WT mice for either early (2-5) or late (6-10) blocks. 

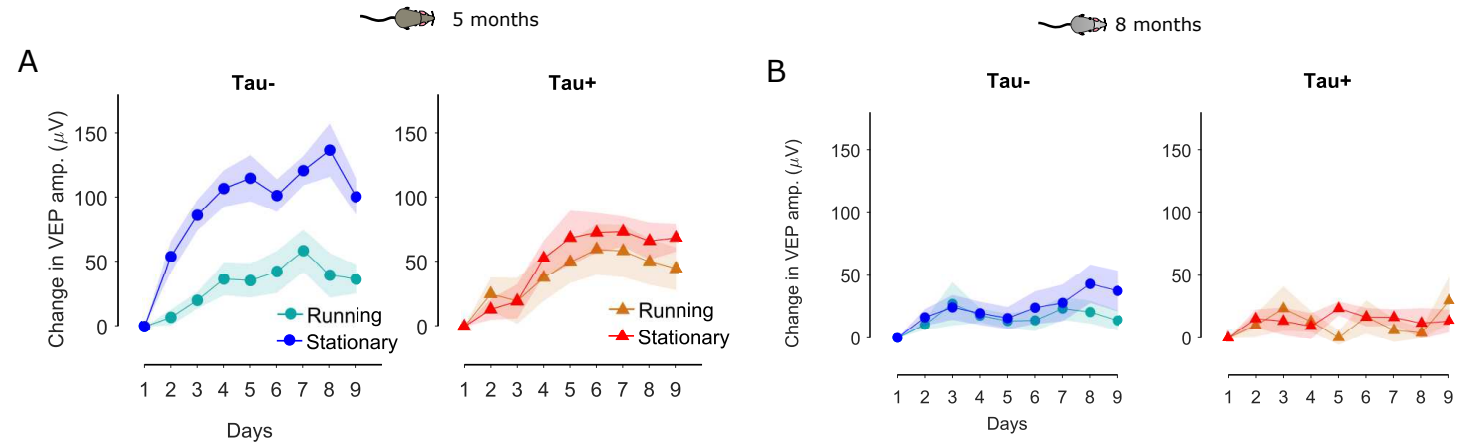

C

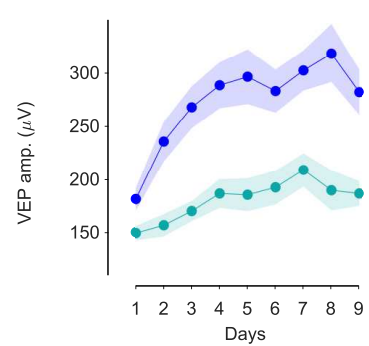

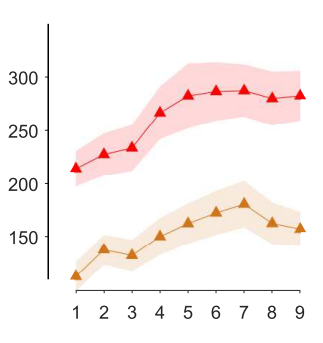

D
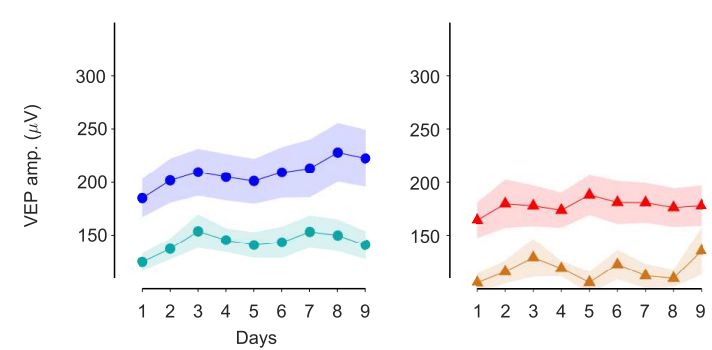

Figure S5: Running reduces the VEP amplitude but does not affect stimulus-response potentiation in either Tau+ or Tau- animals. A. Difference in the VEP amplitude from day 1 for the familiar stimulus for Tau- (left) and Tau+ (right) 5-month old animals over the course of days calculated considering only stationary or running epochs. B. Same as in A for 8-month old animals. C. Unnormalized VEP amplitude as a function of days for 5-month old Tau- (left) and Tau+ (right) mice calculated considering only stationary or running epochs. D. Same as in C for 8-month old animals.

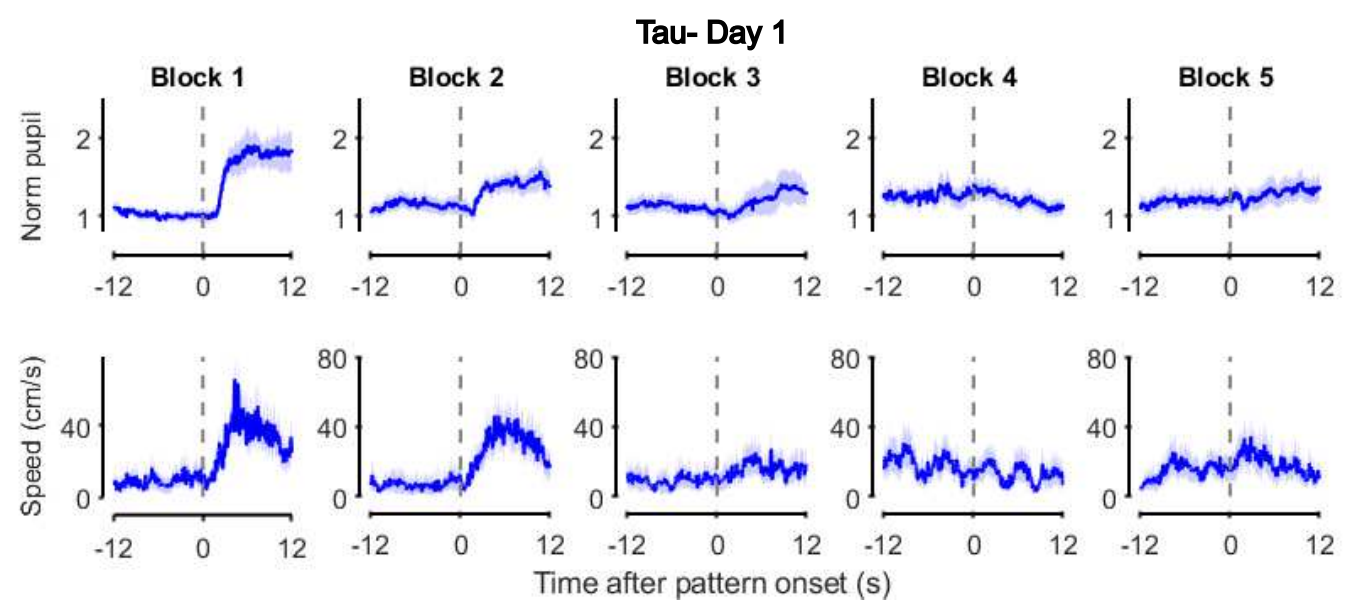

Figure S6: Visual evoked behaviour habituates quickly in Tau- animals. Mean ( \pm SEM) normalized pupil responses (top) and movement speed (bottom) to the onset of the stimulus during the first five blocks of presentation for naive Tau- animals (day 1). 